\title{
ADVANCE SUSTAINABLE INVENTORY MANAGEMENT THROUGH ADVERTISEMENT AND TRADE-CREDIT POLICY
}

\author{
Buddhadev Mandal ${ }^{1}$, Bikash Koli Dey ${ }^{2}$, Sudhansu Khanra ${ }^{3}$ and \\ BISWAJIT SARKAR ${ }^{4, *}$
}

\begin{abstract}
The concept of advanced sustainable inventory management, where demand pattern stock level and advertising dependent under trade-credit policy is taking account in this present study. Optimal credit period and cycle time are the main objective of this advanced system. A developed solution methodology is derived to show the existence of global optimality under optimum credit period and cycle time. The main concern of this advanced system is to maximize the annual total system profit of retailer with finite replenishment rate. Numerical illustration are carry forward for different cases to prove the stainability along with real impact of this model. Sensitive analysis for the key parameters is discussed in sensitivity analysis section along with some real managerial insights.
\end{abstract}

Mathematics Subject Classification. 90B05, 90B06.

Received January 29, 2020. Accepted June 19, 2020.

\section{INTRODUCTION}

In today's competitive business world each and every industry wants more profits, as advertising gives a chance to sell more product, thus every industry invests more in advertising to optimum their profit. Thus, every industry maintains a good advertising team to optimum their profit. This is a strategy to sell maximum items at minimum duration. Due to increases of demand, selling of the items also increased. This advertisement helps to identify products with different facilities, and which one is more reliable among all other products in the market. The advertising increases the probability of successful marketing targets with the fulfill demand of the customers. As a result, maximum investment for advertising gives more profits to any company. In this competitive marketing system, every supplier or manufacturer wants to sells more items to earn more revenues, reminding this most of the suppliers/manufacturer offer a certain delay period to convince the retailer/customer to buy more items. Retailer can sell his/her product before the end of the delay period, and interest earns

Keywords. Sustainable inventory management, advertisement cost, permissible delay-in-payments, shortages, trade-credit policy.

1 Department of Industrial and Systems Engineering, School of Engineering and Sciences, Tecnológico de Monterrey, E. Garza Sada 2501 Sur, C.P. 64849 Monterrey, Nuevo León, Mexico.

2 Department of Industrial Engineering, Hongik University, 72-1 Mapo-Gu, Sangsu-Dong, Seoul 04066, Republic of Korea.

3 Department of Mathematics, Tamralipta Mahavidyalaya, Tamluk, Purba Medinipur 721636, India.

4 Department of Industrial Engineering, Yonsei University, 50 Yonsei-ro, Sinchon-Dong, Seadaemun-gu, Seoul 03722, South Korea.

*Corresponding author: bsbiswajitsarkar@gmail.com 
with revenue. So, the delay-in-payments strategy is one kind of price-discount policy which encourages the retailers/customer to increase their order quantity.

Demand for any inventory system one of the most important issues in today's business industry. For simplification of the model, demand will be considered constant as a basic assumption in many existing literature $[3,6,28]$. But in our daily life it is near about impossible that demand of any product always constant in general. Some times looks of any product attract the customers, so demand of the products can be vary with displayed stock level (DSL) and some times this demand may vary with the time, that is time dependent demand rate, as a result the demand is not constant. To increases the profit, any retailer must have to stocks as much as he can, other wise due to short stock customer can go away from his/her shop. Thus, in any industry DSL have a great impact. Display of inventory is proportional to the sales in retail shop was calculated by Silver and Peterson [49]. In the same direction Mashud et al. [21], Shaikh et al. [48] and Khan et al. [13] developed different inventory model where demand was depend on stock-level. The developed the model for perishable items. More recent, Sana and Chaudhuri [29] developed a volume-flexible stock-dependent demand model. A generalised model for stock dependent was formulated by Shaikh et al. [47] in this model they also considered full backlogging. All research discussed about stock dependent demand model but Sarkar et al. [35] developed a different strategy to find out the optimality for the supplier.

There were different inventory models were developed by different researchers. An advanced stock-dependent inventory model was discussed by Sarkar and Sarkar [33] where deterioration depend on time. This model was extended by Sarkar [31] to a production-inventory model and by considering probabilistic deterioration. In the same year Sarkar et al. [36] developed an inventory model by considering trade-credit policy and price-discount offer. A distribution free continuous-review model with service level constraint was developed by Moon et al. [26]. An integrated inventory model with unequal lot size and variable setup cost was developed by Sarkar et al. [38]. A continuous-review inventory model with controllable lead time and service level constraint was presented by Shin et al. [39]. All researcher developed different advanced inventory model by considering lead time, deterioration, tread-credit etc., but no one concerned about increasing the demand of the product, which directly optimised the profit of the whole system. To increase the demand of a particular product in this research the concept of advertisement policy is adopted along with trade-credit policy, which directly increases the total profit of the system.

Researchers are always busy to developed different type of model of different marketing sector. In today's active social sites and presence of media advertisement for any product has a great impact for any industry. So advertising demand pattern for any industry is one of the great finding for any researcher. It is quite natural that any industry can use the best advertise policy for their product and for that they formulate their best marketing team. Normally the aim of advertising is to awareness of the common people. It inform the general public about the product and influence to the customer to buy their product instead of other, so it compare the difference of product. Any advertisement base on the message which it try to give the general people and what type of media are used. There are different types of media are available for any type of advertisement like as electronic medias, magazines, cable networks, door-to-door sales, newspapers, etc. which implies more demands of the products. Therefore, investing on advertisement definitely incress the demand of any product thus, industries are now invest more on advertisement section to optimum their profit by selling more products. Considering this, Sethi [46] survey a dynamic optimal control model where advertising takes place. Goodwill for any company is one of the key parameter to sell their product, reminding this Tapiero [52] developed the inventory model in which both advertising and goodwill take places in the presence of uncertainty. An advertising model for non-instantaneous deterioration along with trade-credit and preservation technology was developed by Mashud et al. [22,23]. An interesting cooperative advertising collaboration policy for supply chain model was introduced by Sarkar et al. [43]. Recently, an inventory model for stock and advertising demand model was presented by Sana and Chaudhuri [30], where they approach a concentrate investment for advertising as well as increasing the sales effort to maximize the profit. Recently, advertisement dependent demand for two-echelon supply chain model was proposed by Noh et al. [27]. They used Stackelberg game policy to solve the model. There different model for stock dependent demand and advertising dependent demand were separately developed by several 
researchers, but a sustainable inventory model where demand depend both on stock-level and advertisement still not considered by any researcher. In reality, advertisement is one of the best strategy for increase any business industry. Thus, advertisement and stock-level dependent demand is always helpful for any industry to increase their profit, this pioneer attempt is taken in this model.

In this huge competitive market, to increase the sales of any particular product, the supplier made a strategy in which he/she offers a certain fixed time period in that time the retailer pay the amount for the purchased item. This is the trade-credit period, which offered to the retailer by the supplier. There is a chance to the retailers to concentrate their revenue and collect interest with the help of the products during the delay period, whereas a larger interest amount has to be charged if the deposit of amount is not done at the end of the credit period. There is no penalty is charge if the remaining money is paid within the given permissible delay period. In this direction, in response to temporary price/credit incentives Tiwari et al. [54] discussed about retailers pricing credit and some inventory policies for deteriorating items. In the same direction a model with exponential deterioration and partial trade-credit was introduced by Sarkar and Saren [32]. An optimal economic ordering policy is proposed by Sarkar et al. [44] for deteriorating items. Where, they assumed different cases of trade credit period for finite horizon. An EOQ (economic order quantity) model with deteriorating items was deduced by Chang et al. [4], in which the supplier provides a permissible time period to the customer, provided the quantity that he/she will ordered must be larger than or equal to the quantity which he/she predetermined to take. They characterized the optimal solution. More over they developed an easy-to-use algorithm which helps to obtain the optimal order quantity with replenishment time. A permissible delay-in-payments for EOQ models, which was depends on some conditions, is introduced by Chen et al. [5]. A partial tread-credit policy for retailer was proposed by Chung [7]. In this model, he minimised the total cost of the system with optimum order quantity. The concept of two-levels trade-credit was used by Mashud et al. [24]. They consider price-dependent demand for an inventory model along with shortages. A lead time depended ordering cost for multi-stage production model was developed by Kim and Sarkar [20]. Different study about supply chain or integrated inventory were done by different researchers where warranty policy takes a major role in the optimization of total cost or profit $[11,17]$. Taleizadeh et al. [51] developed a production model for multi-product, where delayed payment takes places. In this model they also considered the repair failure and backlogging strategies. To increase the life-time of the deteriorating items preservation technology can helps allot, proved by Iqbal and Sarkar [12]. In long run process a machine definitely produced defective items in out-of-control state. To detect the imperfect items and remove them an inspection is needed Sarkar et al. [37]. Some time inspection error had a great impact on any inventory system, proposed by Khanna et al. [18]. More recent, Dey et al. [8] derived an integrated model where demand is selling price dependent. An integrated model with imperfect production was developed by Sarkar et al. [40]. In the presence of two-level trade-credit policy Soni and Patel [50] derived an integrated inventory model where production rate is variable and demand is price-sensitive. An inventory model with expiration date and pricing decisions was presented by Khan et al. [14] Considering advanced payment policy and trade-credit, different inventory models were formulated by different researchers [15,23,24]. All researcher developed different model for trade-credit or for advertisement policy, but the benefit for advertisement and stock-level dependent demand in the presence of trade-credit for inventory model still not considered. Thus, this model try to fulfill this gap. An author(s) contribution table (Tab. 1), is provided to show the research gaps in this direction.

This model is structured as follows, the next section contains the problem definition along with notation and assumptions. In Section 3, the solution procedure of this model is discussed followed by the mathematical model. Next section consists some numerical examples to elaborate the model. Sensitivity analysis of key parameters and some managerial insights are discussed in Sections 5 and 6. Finally, some concluding remarks with future extension are discussed in conclusion section.

\section{Problem definition, notation, And assumptions}

In this section, one can find the definition of the problem, that is formulated in this current study along with Notation, and assumptions which are considered to developed this model. 
TABLE 1. Research gaps and contributions of previous Author(s).

\begin{tabular}{|c|c|c|c|c|}
\hline Author(s) & Demand & Model & Strategy & $\begin{array}{l}\text { Deterioration } \\
\text { rate }\end{array}$ \\
\hline Shaikh et al. [48] & $\begin{array}{l}\text { Stock } \\
\text { dependent }\end{array}$ & Inventory & $\begin{array}{l}\text { Price } \\
\text { discount }\end{array}$ & Yes \\
\hline Sarkar et al. [35] & $\begin{array}{l}\text { Stock } \\
\text { dependent }\end{array}$ & Inventory & NA & NA \\
\hline Mashud et al. [23] & Inventory-level & Inventory & Backlogging & Yes \\
\hline Chang et al. [4] & $\begin{array}{l}\text { Stock } \\
\text { dependent }\end{array}$ & EOQ & Backlogging & Yes \\
\hline Dey et al. [8] & Selling-price & $\begin{array}{l}\text { Integrated } \\
\text { inventory }\end{array}$ & $\begin{array}{l}\text { Cost } \\
\text { reduction }\end{array}$ & NA \\
\hline Khan et al. [15] & Constant & Inventory & $\begin{array}{l}\text { Advance } \\
\text { payment }\end{array}$ & Yes \\
\hline $\begin{array}{l}\text { Soni and } \\
\text { Patel }[50]\end{array}$ & Constant & $\begin{array}{l}\text { Integrated } \\
\text { inventory }\end{array}$ & Trade-credit & NA \\
\hline Khanra et al. [19] & $\begin{array}{l}\text { Time } \\
\text { dependent }\end{array}$ & Inventory & Trade-credit & NA \\
\hline This paper & $\begin{array}{l}\text { Advertisement \& } \\
\text { stock-level } \\
\text { dependent }\end{array}$ & Inventory & Tread-credit & $\begin{array}{l}\text { Constant function } \\
\text { of on-hand } \\
\text { inventory }\end{array}$ \\
\hline
\end{tabular}

Notes. NA-Not applicable.

\subsection{Problem definition}

Here, an advanced inventory model for single type of item, which has a constant deterioration rate is derived. To illustrate the practical situation, demand is considered as stock-level and advertising dependent. To make the model more sustainable, deterioration rate is considered as the constant function of on-hand inventory. No outstanding payment to the supplier during placing an order along with no interest is to be charged after commencement. Due to large production, shortages are not allowed. A partial trade-credit policy is implied to optimised the total system profit. Finally, the total profit of the system was optimised based on the optimum value of delay period and total cycle time.

\subsection{Notation}

The following notation are use to develop the model.

\begin{tabular}{ll}
\hline Decision variables & \\
\hline$T$ & Length of the cycle (year) \\
$T_{1}$ & Length of time in which inventory reach the zero level (year) \\
\hline Parameters & \\
\hline$d(I(t), t, v)$ & Demand rate function \\
$I(t)$ & Initial inventory at time $t \geq 0$ \\
$\theta$ & Constant deterioration rate of on-hand inventory, $0<\theta<1$ \\
$m$ & permissible delay time in staling the account $($ year $)$ \\
$I_{r}$ & Chargeable interest per rupee investment in stock $(\$ /$ year $)$ \\
$I_{e}$ & Earned interest per rupee investment in a year, $I_{e} \leq I_{r}(\$ /$ year $)$ \\
$c_{h}$ & Inventory holding cost per unit at time $(\$ /$ unit $/$ year $)$ \\
$c_{p}$ & Production cost per unit $(\$ /$ unit $)$ \\
$c_{0}$ & Setup cost per cycle $(\$ /$ setup) \\
$p$ & Selling price per unit $(\$ /$ unit $)$ \\
$v$ & Advertisement cost per cycle $(\$ /$ cycle) \\
\hline
\end{tabular}




\subsection{Assumptions}

The following presumptions are constructed to formulate this model.

(1) The inventory system covers single type of item and the demand rate is a function of stock on display, time and advertisement cost.

(2) No outstanding payment to the supplier during placing an order that is $m<T$ as well as no interest is to be changed after commencement.

(3) The rate of deterioration per unit time is a constant function of on-hand inventory. i.e., Deterioration depends on the total on-hand inventory. If inventory is large, then deterioration rate also increased.

(4) Lead time is the time gap between placing an order and receiving the order, which is considered as negligible in this model, i.e., replenishment occurs instantly just after placing the order.

(5) Production rate always greater then demand so, shortage are not allowed.

(6) The planning period is infinite length and the ending inventory levels are zero.

\section{Model FORMulation}

The demand pattern for the model depend on stock level and advertisement and that is $D(I(t), t, v)=$ $\alpha . I(t)+\beta(a t+b)+x v^{y}$ where $x$ and $y$ are empirically determined constants, which indicate the effectiveness of advertising $0 \leq y \leq 1 . \alpha, \beta, a, b$ are the scaling parameters. $x=0$ indicates that demand is independent of advertising expenditure. For any $y>0$, the increase value of $y$, is more effective in the advertising. Thus, the basic differential equation is

$$
\frac{\mathrm{d} I(t)}{\mathrm{d} t}=-\alpha \theta I(t)-\beta(a t+b)-x v^{y}, \quad 0 \leq t \leq T
$$

with $I(0)=Q_{0}$ and $I(T)=0$.

The solution of equation (3.1) in $0 \leq t \leq T$ is

$$
\begin{aligned}
I(t)= & -\beta\left(\frac{a t}{\alpha \theta}-\frac{a}{\alpha^{2} \theta^{2}}+\frac{b}{\alpha \theta}\right)-\frac{x v^{y}}{\alpha \theta} \\
& +\left\{\left(\beta\left(\frac{a T}{\alpha \theta}-\frac{a}{\alpha^{2} \theta^{2}}+\frac{b}{\alpha \theta}\right)+\frac{x v^{y}}{\alpha \theta}\right) e^{\alpha \theta(T-t)}\right\} .
\end{aligned}
$$

As the delay period has a great impact on demand, so, distributor or whole-seller allowed a delay period $m$ for the retailer or customers. So, depend on the value of $m, T_{1}$, and $T$, three different models developed in this paper.

Case 1. Let $m \leq T_{1} \leq T$.

In Figure 1, inventory versus with the time for model I, $m \leq T_{1}<T$, that is the length of the total planing horizon is grater then the credit period. In this situation buyer can use the revenue of sales to assembled the delay charge with an annual rate $I_{e}$ in $[0, m]$. The interest earned $E_{1}$ is given by

$$
\begin{aligned}
E_{1}= & p I_{e} \int_{0}^{m} t D(t) \mathrm{d} t \\
= & p I_{e} \int_{0}^{m} t \cdot\left\{\alpha \cdot I(t)+\beta(a t+b)+x v^{y}\right\} \mathrm{d} t \\
= & p I_{e}\left\{-\beta\left(\frac{a m^{3}}{3 \theta}-\frac{a m^{2}}{2 \alpha \theta^{2}}+\frac{b m^{2}}{2 \theta}\right)+\frac{x v^{y}}{2} m\left(1-\frac{1}{\theta}\right)+\frac{a \beta m^{3}}{3}+\frac{b \beta}{2} m^{2}\right\} \\
& +p I_{e}\left\{\beta\left(\frac{a T m^{2}}{2 \theta}-\frac{a m^{2}}{2 \alpha \theta^{2}}+\frac{b m^{2}}{\theta}\right)+\frac{x v^{y}}{\theta}\right\}\left\{e^{\theta T}-(1+m) e^{\theta(T-m)}\right\} .
\end{aligned}
$$




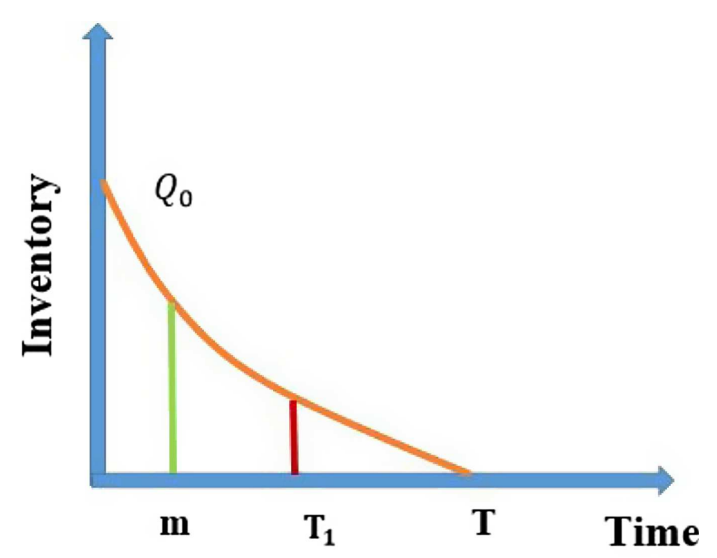

FiguRE 1. Inventory level versus time for Model I $m \leq T_{1}<T$.

After credit-period, the stock which was not sold is to be economics with an annual rate $I_{r}$ so the payable interest $I$ is as follows

$$
\begin{aligned}
I= & p I_{r} \int_{m}^{T_{1}} I(t) \mathrm{d} t \\
= & -p I_{r} \frac{\beta}{2 \alpha \theta}\left(T_{1}^{2}-m^{2}\right)+p I_{r}\left\{\beta\left(\frac{a}{\alpha^{2} \theta^{2}}-\frac{b}{\alpha \theta}\right)\left(T_{1}-m\right)-\frac{x v^{y}}{\alpha \theta}\left(T_{1}-m\right)\right\} \\
& +p I_{r}\left\{\beta\left(\frac{a T}{\alpha \theta}-\frac{a}{\alpha^{2} \theta^{2}}+\frac{b}{\alpha \theta}\right)+\frac{x v^{y}}{\alpha \theta}\right\}\left\{e^{\alpha \theta(T-m)}-e^{\alpha \theta\left(T-T_{1}\right)}\right\}
\end{aligned}
$$

Therefore, the average profit is

$$
\begin{aligned}
Z_{1}= & \frac{1}{T}\left[\left(p-c_{p}\right) \int_{0}^{T} D(I(t), t, v) \mathrm{d} t-c_{0}-v-c_{h} \int_{0}^{T} I(t) \mathrm{d} t-\theta c_{p} \int_{T_{1}}^{T} I(t) \mathrm{d} t-I+E_{1}\right] \\
= & \frac{1}{T}\left[\left(p-c_{p}\right) \int_{0}^{T}\left\{\beta(a t+b)+x v^{y}\right\} \mathrm{d} t+E_{1}-c_{0}-v-I\right. \\
& \left.+\left(p-c_{p}-c_{h}\right)(1+\alpha) \int_{0}^{T} I(t) \mathrm{d} t-\theta c_{p} \int_{T_{1}}^{T} I(t) \mathrm{d} t-E_{1}\right] \\
= & \frac{1}{T}\left[\left(p-c_{p}\right)\left\{\beta\left(\frac{a T^{2}}{2}+b T\right)+x V^{y} T\right\}-\left(c_{0}+v\right)+p I_{e}\left\{-\alpha \beta\left(\frac{a m^{3}}{3 \theta}-\frac{m^{2}}{2 \theta}+\frac{b m^{2}}{2 \theta}\right)\right.\right. \\
& \left.+\frac{x v^{y}}{2} m\left(\frac{1}{\theta}+1\right)+\frac{a \beta m^{2}}{3}+\frac{b \beta}{2} m^{2}\right\}+p I_{e}\left\{\beta\left(\frac{a T}{\theta}-\frac{1}{\theta}+\frac{b}{\theta}\right)-\frac{x v^{y}}{\theta}\right\}\left\{e^{\theta T}-(1+m) e^{\theta(T-m)}\right\} \\
& +\left(p-c_{p}-c_{h}\right)(1-\alpha)\left\{-\beta\left(\frac{a T^{2}}{2 \theta}-\frac{T^{2}}{\theta^{2}}+\frac{b T}{\theta}\right)+\frac{x v^{y}}{\theta} T+\beta\left(\frac{a T}{\theta}-\frac{1}{\theta^{2}}+\frac{b}{\theta}\right)-\frac{x v^{y}}{\theta}\right\} e^{\theta T} \\
& +p I_{r} \frac{\alpha \beta}{2 \theta}\left(T_{1}^{2}-m^{2}\right)-p I_{r}\left\{\beta\left(\frac{1}{\theta^{2}}-\frac{b}{\theta}\right)+\frac{x v^{y}}{\theta}\right\}\left(T_{1}-m\right) \\
& -p I_{r}\left\{\beta\left(\frac{a T}{\theta}-\frac{1}{\theta^{2}}+\frac{b}{\theta}\right)-\frac{x v^{y}}{\theta}\right\}\left\{e^{\theta(T-m)}-e^{\theta\left(T-T_{1}\right)}\right\}+\theta c_{p} \frac{a \beta}{2 \theta}\left(T^{2}-T_{1}^{2}\right)
\end{aligned}
$$




$$
\left.-\theta c_{p}\left\{\beta\left(\frac{1}{\theta^{2}}-\frac{b}{\theta}\right)+\frac{x v^{y}}{\theta}\right\}\left(T-T_{1}\right)-\left\{\beta\left(\frac{a T}{\theta}-\frac{1}{\theta^{2}}+\frac{b}{\theta}\right)-\frac{x v^{y}}{\theta}\right\} e^{\theta\left(T-T_{1}\right)}\right] .
$$

The purpose is to achieve the maximum average profit per unit time.

The following theorem was used from Khanra et al. [19].

Theorem 3.1. If a function $U\left(T_{1}, T\right)=\frac{1}{T} W\left(T_{1}, T\right)$ where $W\left(T_{1}, T\right)$ is a continuously partial differentiable function of $T_{1}$ and $T$ of second order then $U\left(T_{1}, T\right)$ is maximum at $T_{1}=T_{1}^{*}, T=T^{*}$ if $d^{2} W\left(T_{1}, T\right)$ is positive definite i.e., if

$$
\left|\begin{array}{ll}
\frac{\partial^{2} W\left(T_{1}, T\right)}{\partial T_{1}^{2}} & \frac{\partial^{2} W\left(T_{1}, T\right)}{\partial T_{1} \partial T} \\
\frac{\partial^{2} W\left(T_{1}, T\right)}{\partial T_{1} \partial T} & \frac{\partial^{2} W\left(T_{1}, T\right)}{\partial T^{2}}
\end{array}\right|>0 \quad \text { and } \quad \frac{\partial^{2} W\left(T_{1}, T\right)}{\partial T_{1}^{2}}<0 \quad \text { or } \quad \frac{\partial^{2} W\left(T_{1}, T\right)}{\partial T^{2}}<0 .
$$

[See Appendix A for proof].

Lemma 3.2. $Z_{1}\left(T_{1}, T\right)$ has the maximum value at the optimised values of $T$ and $T_{1}$, that satisfy following equations

(ii)

$$
\begin{aligned}
p I_{r} & \left\{\frac{\alpha \beta}{\theta} T_{1}-\beta\left(\frac{1}{\theta^{2}}-\frac{b}{\theta}-\frac{x v^{y}}{\theta}\right)\right\}-\left\{\beta\left(\frac{a T}{\theta}-\frac{1}{\theta^{2}}+\frac{b}{\theta}\right)-\frac{x v^{y}}{\theta}\right\} e^{\theta\left(T-T_{1}\right)} \\
& -\theta c_{p}\left\{\frac{\alpha \beta}{\theta} T_{1}-\beta\left(\frac{1}{\theta^{2}}-\frac{b}{\theta}\right)-\frac{x v^{y}}{\theta}\right\} \\
& +\theta c_{p}\left\{\beta\left(\frac{a T}{\theta}-\frac{1}{\theta^{2}}+\frac{b}{\theta}\right)-\frac{x v^{y}}{\theta}\right\} e^{\theta\left(T-T_{1}\right)}=0 \\
(p & \left.-c_{p}\right)\left\{\beta(a T+b)+\frac{x v^{y}}{\theta}\right\}+p I_{e}\left\{\beta\left(\frac{a T}{\theta}-\frac{1}{\theta}+\frac{b}{\theta}\right)-\frac{x v^{y}}{\theta}\right\} \\
& \times\left\{e^{\theta T}-(1+m) e^{\theta(T-m)}\right\}+\frac{p I_{e} \alpha \beta}{\theta}\left\{e^{\theta T}-(1+m) e^{\theta(T-m)}\right\} \\
& +\left(p-c_{p}-c_{h}\right)(1+\alpha)\left\{-\beta\left(\frac{a T}{\theta}-\frac{1}{\theta^{2}}+\frac{b}{\theta}\right)+\frac{x v^{y}}{\theta}\right\} \\
& +\left(p-c_{p}-c_{h}\right)(1+\alpha)\left\{\beta\left(\frac{a T}{\theta}-\frac{1}{\theta^{2}}+\frac{b}{\theta}\right)-\frac{x v^{y}}{\theta}\right\} e^{\theta T} \\
& +\left(p-c_{p}-c_{h}\right)(1+\alpha) \frac{a \beta}{\theta} e^{\theta T}-p \theta I_{r}\left\{\beta\left(\frac{a T}{\theta}-\frac{1}{\theta^{2}}+\frac{b}{\theta}\right)-\frac{x v^{y}}{\theta}\right\}\left\{e^{\theta(T-m)}-e^{\theta\left(T-T_{1}\right)}\right\} \\
& -p \alpha \beta I_{r}\left\{e^{\theta(T-m)}-e^{\theta\left(T-T_{1}\right)}\right\}+\alpha \beta c_{p} T-\theta c_{p}\left\{\beta\left(\frac{1}{\theta^{2}}-\frac{b}{\theta}\right)+\frac{x v^{y}}{\theta}\right\} \\
& +a \beta c_{p} e^{\theta\left(T-T_{1}\right)}+\theta^{2} c_{p}\left\{\beta\left(\frac{a T}{\theta}-\frac{1}{\theta^{2}}+\frac{b}{\theta}\right)-\frac{x v^{y}}{\theta}\right\} e^{\theta\left(T-T_{1}\right)}
\end{aligned}
$$

provided the following sufficient conditions are satisfied

$$
\begin{gathered}
\text { (i) } \frac{p I_{r} \alpha \beta}{\theta}-p I_{r}\left\{\beta\left(\frac{a T}{\theta}-\frac{1}{\theta^{2}}+\frac{b}{\theta}\right)-\frac{x v^{y}}{\theta}\right\} e^{\theta\left(T-T_{1}\right)} \\
>\alpha \beta c_{p}-p I_{r} \theta c_{p}\left\{\beta\left(\frac{a T}{\theta}-\frac{1}{\theta^{2}}+\frac{b}{\theta}\right)-\frac{x v^{y}}{\theta}\right\} e^{\theta\left(T-T_{1}\right)} \\
\text { (ii) }\left(p-c_{p}\right) \alpha \beta+p I_{e} \theta\left\{\beta\left(\frac{a T}{\theta}-\frac{1}{\theta^{2}}+\frac{b}{\theta}\right)-\frac{x v^{y}}{\theta}\right\}\left\{e^{\theta T}-(1+m) e^{\theta(T-m)}\right\}
\end{gathered}
$$




$$
\begin{aligned}
& +\left(p-c_{p}-c_{h}\right)(1+\alpha)\left(\frac{1+\theta}{\theta}\right) a \beta e^{\theta T} \\
& +\theta\left(p-c_{p}-c_{h}\right)(1+\alpha)\left\{\beta\left(\frac{a T}{\theta}-\frac{1}{\theta^{2}}+\frac{b}{\theta}\right)-\frac{x v^{y}}{\theta}\right\} e^{\theta T}+a \beta c_{p} \\
& >\left(p-c_{p}-c_{h}\right)(1+\alpha) \frac{\alpha \beta}{\theta}+p a \beta I_{r} \frac{1+\theta}{\theta}\left\{e^{\theta(T-m)}-e^{\theta\left(T-T_{1}\right)}\right\} \\
& +p \theta^{2} I_{r}\left\{\beta\left(\frac{a T}{\theta}-\frac{1}{\theta^{2}}+\frac{b}{\theta}\right)-\frac{x v^{y}}{\theta}\right\}\left\{e^{\theta(T-m)}-e^{\theta\left(T-T_{1}\right)}\right\}
\end{aligned}
$$

$$
\begin{aligned}
\theta c_{p} & \left\{\beta\left(\frac{a T}{\theta}-\frac{1}{\theta^{2}}+\frac{b}{\theta}\right)-\frac{x v^{y}}{\theta}\right\} e^{\theta\left(T-T_{1}\right)}+a \beta c_{p} e^{\theta\left(T-T_{1}\right)} \\
& >p I_{r} \theta\left\{\beta\left(\frac{a T}{\theta}-\frac{1}{\theta^{2}}+\frac{b}{\theta}\right)-\frac{x v^{y}}{\theta}\right\} e^{\theta\left(T-T_{1}\right)}+\frac{a \beta p I_{r}}{\theta} e^{\theta\left(T-T_{1}\right)} .
\end{aligned}
$$

[See Appendix B for proof].

Case 2. $T_{1}<m<T$.

Figure 2 shows that no interest should be paid by the customer, but earns some interest at an annual rate $I_{e}$, in the time period $[0, m]$, that earned interest is $E_{2}$ (see [19]) given by

$$
\begin{aligned}
E_{2}= & p I_{e} \int_{0}^{T_{1}} t D(t) \mathrm{d} t+p I_{e}\left(m-T_{1}\right) \int_{0}^{T_{1}} D(t) \mathrm{d} t \\
= & p I_{e}\left\{-\alpha \beta\left(\frac{a T_{1}^{3}}{3 \theta}-\frac{T_{1}^{2}}{2 \theta^{2}}+\frac{b T_{1}^{2}}{2 \theta}\right)+\frac{x v^{y}}{2} T_{1}^{2}\left(\frac{1}{\theta}+1\right)\right. \\
& \left.+\frac{a \beta T_{1}^{2}}{3}+\frac{b \beta}{2} T_{1}^{2}\right\}+p I_{e}\left\{\beta\left(\frac{a T}{\theta}-\frac{1}{\theta^{2}}+\frac{b}{\theta}\right)-\frac{x v^{y}}{\theta}\right\}\left\{e^{\theta T}-\left(1+T_{1}\right) e^{\theta\left(T-T_{1}\right)}\right\} \\
& +p I_{e}\left(m-T_{1}\right)\left\{-\alpha \beta\left(\frac{a T_{1}^{3}}{3 \theta}-\frac{T_{1}}{2 \theta^{2}}+\frac{b T_{1}^{2}}{2 \theta}\right)+\frac{x v^{y}}{2} T_{1}\left(\frac{1}{\theta}+1\right)+\frac{a \beta T_{1}^{2}}{3}+\frac{b \beta}{2} T_{1}\right\} \\
& +p i_{e}\left(m-T_{1}\right)\left\{\beta\left(\frac{a T}{\theta}-\frac{1}{\theta^{2}}+\frac{b}{\theta}\right)-\frac{x v^{y}}{\theta}\right\}\left\{e^{\theta T}-e^{\theta\left(T-T_{1}\right)}\right\} .
\end{aligned}
$$

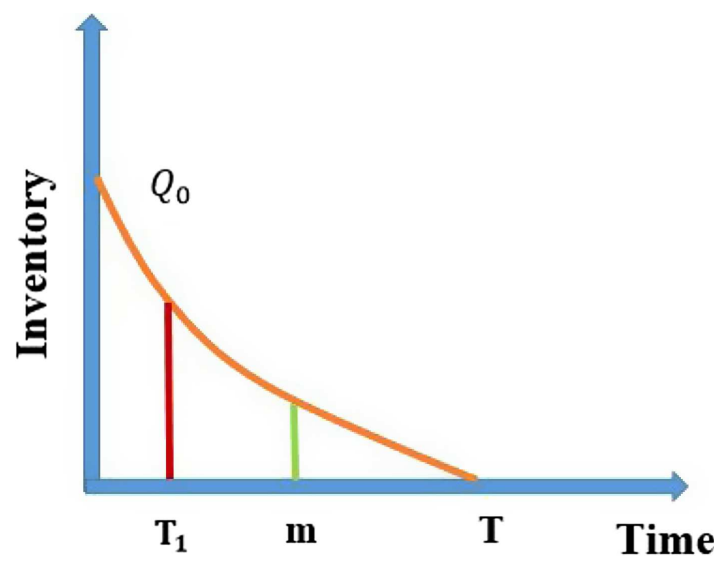

Figure 2. Inventory level versus time for Model II $T_{1}<m<T$. 
As all items are sold in this case so there is no payable interest for this case. Therefore, the total average profit in this case is as follows

$$
\begin{aligned}
Z_{2}= & \frac{1}{T}\left[\left(P-c_{P}\right) \int_{0}^{T} D(I(t), t, v) \mathrm{d} t-c_{0}-v-c_{h} \int_{0}^{T} I(t) \mathrm{d} t-\theta c_{p} \int_{T_{1}}^{T} I(t) \mathrm{d} t+E_{2}\right] \\
= & \frac{1}{T}\left[\left(p-c_{p}\right)\left\{\beta\left(\frac{a T^{2}}{2}+b T\right)+x v^{y} T\right\}-\left(c_{0}+V\right)\right. \\
& +p I_{e}\left\{-\alpha \beta\left(\frac{a T_{1}^{3}}{3 \theta}-\frac{T_{1}^{2}}{2 \theta^{2}}+\frac{b T_{1}^{2}}{2 \theta}\right)+\frac{x v^{y}}{2} T_{1}^{2}\left(\frac{1}{\theta}+1\right)+\frac{a \beta T_{1}^{2}}{3}+\frac{b \beta}{2} T_{1}^{2}\right\} \\
& +p I_{e}\left\{\beta\left(\frac{a T}{\theta}-\frac{1}{\theta^{2}}+\frac{b}{\theta}\right)-\frac{x v^{y}}{\theta}\right\}\left\{e^{\theta T}-\left(1+T_{1}\right) e^{\theta\left(T-T_{1}\right)}\right\} \\
& +p I_{e}\left(m-T_{1}\right)\left\{-\alpha \beta\left(\frac{a T_{1}^{2}}{3 \theta}-\frac{T_{1}}{2 \theta^{2}}+\frac{b T_{1}}{2 \theta}\right)+\frac{x v^{y}}{2} T_{1}\left(\frac{1}{\theta}+1\right)+\frac{a \beta T_{1}}{3}+\frac{b \beta}{2} T_{1}\right\} \\
& +p I_{e}\left(m-T_{1}\right)\left\{\beta\left(\frac{a T}{\theta}-\frac{1}{\theta^{2}}+\frac{b}{\theta}\right)-\frac{x v^{y}}{\theta}\right\}\left\{e^{\theta T}-e^{\theta\left(T-T_{1}\right)}\right\} \\
& +\left(p-c_{p}-c_{h}\right)(1+\alpha)\left\{-\beta\left(\frac{a T^{2}}{\theta}-\frac{T}{\theta^{2}}+\frac{b T}{\theta}\right)+\frac{x v^{y}}{\theta} T\right\} \\
& +\left(p-c_{p}-c_{h}\right)(1+\alpha)\left\{\beta\left(\frac{a T}{\theta}-\frac{1}{\theta^{2}}+\frac{b}{\theta}\right)-\frac{x v^{y}}{\theta}\right\} e^{\theta T}+\theta c_{p} \frac{a \beta}{2 \theta}\left(T^{2}-T_{1}^{2}\right) \\
& -\theta c_{p}\left\{\beta\left(\frac{1}{\theta^{2}}-\frac{b}{\theta}\right)+\frac{x v^{y}}{\theta}\right\}\left(T-T_{1}\right) \\
& \left.-\theta c_{p}\left\{\beta\left(\frac{a T}{\theta}-\frac{1}{\theta^{2}}+\frac{b}{\theta}\right)-\frac{x v^{y}}{\theta}\right\} e^{\theta\left(T-T_{1}\right)}\right] .
\end{aligned}
$$

Lemma 3.3. $Z_{2}\left(T_{1}, T\right)$ attaints maximum value for the optimised value of $T$ and $T_{1}$ that also satisfy the following equations

$$
\begin{aligned}
p I_{e} & \left\{-\alpha \beta\left(\frac{a T_{1}^{2}}{\theta}-\frac{T_{1}}{\theta^{2}}+\frac{b T_{1}}{\theta}\right)+\frac{x v^{y}}{2} T_{1}\left(\frac{1}{\theta}+1\right)\right. \\
& \left.+a \beta T_{1}^{2}+b \beta T_{1}\right\}-p \theta I_{e}\left\{\beta\left(\frac{a T}{\theta}-\frac{1}{\theta^{2}}+\frac{b}{\theta}\right)-\frac{x v^{y}}{\theta}\right\}\left\{\left(1+T_{1}\right) e^{\theta\left(T-T_{1}\right)}\right\} \\
& -p I_{e}\left\{\beta\left(\frac{a T}{\theta}-\frac{1}{\theta^{2}}+\frac{b}{\theta}\right)-\frac{x v^{y}}{\theta}\right\} e^{\theta\left(T-T_{1}\right)}-p I_{e}\left\{-\alpha \beta\left(\frac{a T_{1}^{2}}{\theta}-\frac{T_{1}}{\theta^{2}}+\frac{b T_{1}}{\theta}\right)+\frac{x v^{y}}{\theta} T_{1}\right\} \\
& +p I_{e}\left(m-T_{1}\right)\left\{-\alpha \beta\left(\frac{a T_{1}}{\theta}-\frac{1}{\theta^{2}}+\frac{b}{\theta}\right)+\frac{x v^{y}}{\theta}\right\} \\
& -p I_{e}\left\{\beta\left(\frac{a T}{\theta}-\frac{1}{\theta^{2}}+\frac{b}{\theta}\right)-\frac{x v^{y}}{\theta}\right\}\left\{e^{\theta T}-e^{\theta\left(T-T_{1}\right)}\right\} \\
& -p \theta I_{e}\left\{\beta\left(\frac{a T}{\theta}-\frac{1}{\theta^{2}}+\frac{b}{\theta}\right)-\frac{x v^{y}}{\theta}\right\} e^{\theta\left(T-T_{1}\right)}-a \beta c_{p} T_{1}+\theta c_{p}\left\{\beta\left(\frac{1}{\theta^{2}}-\frac{b}{\theta}\right)+\frac{x v^{y}}{\theta}\right\} \\
& +\theta^{2} c_{p}\left\{\beta\left(\frac{a T}{\theta}-\frac{1}{\theta^{2}}+\frac{b}{\theta}\right)-\frac{x v^{y}}{\theta}\right\} e^{\theta\left(T-T_{1}\right)}=0
\end{aligned}
$$


(ii)

$$
\begin{aligned}
(p & \left.-c_{p}\right)\left\{\beta(a T+b)+x v^{y}\right\}+p \theta I_{e}\left\{\beta\left(\frac{a T}{\theta}-\frac{1}{\theta^{2}}+\frac{b}{\theta}\right)-\frac{x v^{y}}{\theta}\right\} \\
& \times\left\{e^{\theta T}-\left(1-T_{1}\right) e^{\theta\left(T-T_{1}\right)}\right\}+\frac{a \beta p I_{e}}{\theta}\left\{e^{\theta T}-\left(1-T_{1}\right) e^{\theta\left(T-T_{1}\right)}\right\} \\
& +\left(m-T_{1}\right) p \theta I_{e}\left\{\beta\left(\frac{a T}{\theta}-\frac{1}{\theta^{2}}+\frac{b}{\theta}\right)-\frac{x v^{y}}{\theta}\right\}\left\{e^{\theta T}-\left(1-T_{1}\right) e^{\theta\left(T-T_{1}\right)}\right\} \\
& +\left(m-T_{1}\right) \frac{a \beta p I_{e}}{\theta}\left\{e^{\theta T}-\left(1-T_{1}\right) e^{\theta\left(T-T_{1}\right)}\right\} \\
& +\left(p-c_{p}-c_{h}\right)(1+\alpha)\left\{-\beta\left(\frac{a T}{\theta}-\frac{1}{\theta^{2}}+\frac{b}{\theta}\right)+\frac{x v^{y}}{\theta}\right\} \\
& +\theta\left(p-c_{p}-c_{h}\right)(1+\alpha)\left\{-\beta\left(\frac{a T}{\theta}-\frac{1}{\theta^{2}}+\frac{b}{\theta}\right)+\frac{x v^{y}}{\theta}\right\} e^{\theta T} \\
& +\left(p-c_{p}-c_{h}\right)(1+\alpha) \frac{a \beta}{\theta}+a \beta c_{p} T-\theta c_{p}\left\{\beta\left(\frac{1}{\theta^{2}}-\frac{b}{\theta}\right)+\frac{x v^{y}}{\theta}\right\} \\
& -\theta^{2} c_{p}\left\{\beta\left(\frac{a T}{\theta}-\frac{1}{\theta^{2}}+\frac{b}{\theta}\right)-\frac{x v^{y}}{\theta}\right\} e^{\theta\left(T-T_{1}\right)}-a \beta c_{p} e^{\theta\left(T-T_{1}\right)}=0
\end{aligned}
$$

provided the following sufficient conditions are satisfied

$$
\begin{aligned}
& p I_{e}\left\{-\alpha \beta\left(\frac{2 a T_{1}}{\theta}-\frac{1}{\theta^{2}}+\frac{b}{\theta}\right)+\frac{x v^{y}}{2}\left(\frac{1}{\theta}+1\right)+2 a \beta T_{1}+b \beta\right\} \\
& -p I_{e}\left\{\beta\left(\frac{a T}{\theta}-\frac{1}{\theta^{2}}+\frac{b}{\theta}\right)-\frac{x v^{y}}{\theta}\right\} e^{\theta\left(T-T_{1}\right)} \\
& +p \theta I_{e}\left\{\beta\left(\frac{a T}{\theta}-\frac{1}{\theta^{2}}+\frac{b}{\theta}\right)-\frac{x v^{y}}{\theta}\right\}\left(1+T_{1}\right) e^{\theta\left(T-T_{1}\right)} \\
& -2 p I_{e}\left\{-\alpha \beta\left(\frac{2 a T_{1}}{\theta}-\frac{1}{\theta^{2}}+\frac{b}{\theta}\right)+\frac{x v^{y}}{\theta}\right\}-\frac{a \alpha \beta p I_{e}}{\theta}\left(m-T_{1}\right) \\
& -p I_{e}\left(m-T_{1}\right)\left\{-\alpha \beta\left(\frac{a T_{1}}{\theta}-\frac{1}{\theta^{2}}+\frac{b}{\theta}\right)+\frac{x v^{y}}{\theta}\right\}-p \theta I_{e}\left\{\beta\left(\frac{a T}{\theta}-\frac{1}{\theta^{2}}+\frac{b}{\theta}\right)\right. \\
& \left.-\frac{x v^{y}}{\theta}\right\} e^{\theta\left(T-T_{1}\right)}-p I_{e}\left\{\beta\left(\frac{a T}{\theta}-\frac{1}{\theta^{2}}+\frac{b}{\theta}\right)-\frac{x v^{y}}{\theta}\right\} e^{\theta\left(T-T_{1}\right)} \\
& -p \theta^{2} I_{e}\left(m-T_{1}\right)\left\{\beta\left(\frac{a T}{\theta}-\frac{1}{\theta^{2}}+\frac{b}{\theta}\right)-\frac{x v^{y}}{\theta}\right\} e^{\theta\left(T-T_{1}\right)} a \beta c_{p} \\
& -\theta^{3} c_{p}\left\{\beta\left(\frac{a T}{\theta}-\frac{1}{\theta^{2}}+\frac{b}{\theta}\right)-\frac{x v^{y}}{\theta}\right\} e^{\theta\left(T-T_{1}\right)}=0 \\
& \text { (ii) } \quad\left(p-c_{p}\right) a \beta+p \theta^{2} I_{e}\left\{\beta\left(\frac{a T}{\theta}-\frac{1}{\theta^{2}}+\frac{b}{\theta}\right)-\frac{x v^{y}}{\theta}\right\}\left\{e^{\theta T}-\left(1+T_{1}\right) e^{\theta\left(T-T_{1}\right)}\right\} \\
& +\frac{a \beta p I_{e}}{\theta}\left\{e^{\theta T}-\left(1+T_{1}\right) e^{\theta\left(T-T_{1}\right)}\right\}+\left(m-T_{1}\right) p \theta^{2} I_{e}\left\{\beta\left(\frac{a T}{\theta}-\frac{1}{\theta^{2}}+\frac{b}{\theta}\right)\right. \\
& \left.-\frac{x v^{y}}{\theta}\right\}\left\{e^{\theta T}-\left(1+T_{1}\right) e^{\theta\left(T-T_{1}\right)}\right\}+2\left(m-T_{1}\right) a \beta p I_{e}\left\{e^{\theta T}-e^{\theta\left(T-T_{1}\right)}\right\} \\
& +\theta^{2}\left(p-c_{p}-c_{h}\right)(1+\alpha)\left\{\beta\left(\frac{a T}{\theta}-\frac{1}{\theta^{2}}+\frac{b}{\theta}\right)+\frac{x v^{y}}{\theta}\right\} e^{\theta T} \\
& +\theta\left(p-c_{p}-c_{h}\right)(1+\alpha) \frac{a \beta}{\theta} e^{\theta T}+\theta\left(p-c_{p}-c_{h}\right)(1+\alpha) a \beta e^{\theta T}+a \beta c_{p}
\end{aligned}
$$




$$
\begin{aligned}
& >\theta\left(p-c_{p}-c_{h}\right)(1+\alpha) \frac{a \beta}{\theta}+\theta^{3} c_{p}\left\{\beta\left(\frac{a T}{\theta}-\frac{1}{\theta^{2}}+\frac{b}{\theta}\right)-\frac{x v^{y}}{\theta}\right\} e^{\theta\left(T-T_{1}\right)} \\
- & 2 a \beta \theta c_{p} e^{\theta\left(T-T_{1}\right)}\left(1+m+\theta c_{P}\right) p I_{e}\left\{\beta\left(\frac{a T}{\theta}-\frac{1}{\theta^{2}}+\frac{b}{\theta}\right)-\frac{x v^{y}}{\theta}\right\} e^{\theta\left(T-T_{1}\right)} \\
& +\left(\theta c_{p}-1\right) \frac{a \beta p I_{e}}{\theta} e^{\theta\left(T-T_{1}\right)}>p I_{e}\left\{\beta\left(\frac{a T}{\theta}-\frac{1}{\theta^{2}}+\frac{b}{\theta}\right)-\frac{x v^{y}}{\theta}\right\} e^{\theta T}
\end{aligned}
$$

[See Appendix C for proof].

Case 3. Let $m \geq T$.

In this case, from Figure 3, some interest was earned by the customer from the sales revenue and this should be earned upto the permissible delay-period. For this time interval $[0, T]$ no interest should be payable.

$$
\begin{aligned}
p I_{e} \int_{0}^{T_{1}} t D(t)= & p I_{e}\left\{-\alpha \beta\left(\frac{a T_{1}^{3}}{3 \theta}-\frac{T_{1}^{2}}{2 \theta}+\frac{b T_{1}^{2}}{2 \theta}\right)+\frac{x v^{y}}{2} T_{1}^{2}\left(\frac{1}{\theta}+1\right)+\frac{a \beta T_{1}^{2}}{3}+\frac{b \beta}{2} T_{1}^{2}\right\} \\
& +p I_{e}\left\{\beta\left(\frac{a T}{\theta}-\frac{1}{\theta}+\frac{b}{\theta}\right)-\frac{x v^{y}}{\theta}\right\}\left\{e^{\theta T}-\left(1+T_{1}\right) e^{\theta\left(T-T_{1}\right)}\right\} .
\end{aligned}
$$

Interest earned for the permissible delay $\left[T_{1}, m\right]$ is

$$
\begin{aligned}
p I_{e} \int_{0}^{T_{1}}\left(m-T_{1}\right) D(t) \mathrm{d} t= & p I_{e}\left(m-T_{1}\right)\left\{-\alpha \beta\left(\frac{a T_{1}^{2}}{3 \theta}-\frac{T_{1}}{2 \theta}+\frac{b T_{1}}{2 \theta}\right)+\frac{x v^{y}}{2} T_{1}\left(\frac{1}{\theta}+1\right)\right. \\
& \left.+\frac{a \beta T_{1}}{3}+\frac{b \beta}{2} T_{1}\right\}+p I_{e}\left(m-T_{1}\right)\left\{\beta\left(\frac{a T}{\theta}-\frac{1}{\theta}+\frac{b}{\theta}\right)-\frac{x v^{y}}{\theta}\right\}\left\{e^{\theta T}-e^{\theta\left(T-T_{1}\right)}\right\} .
\end{aligned}
$$

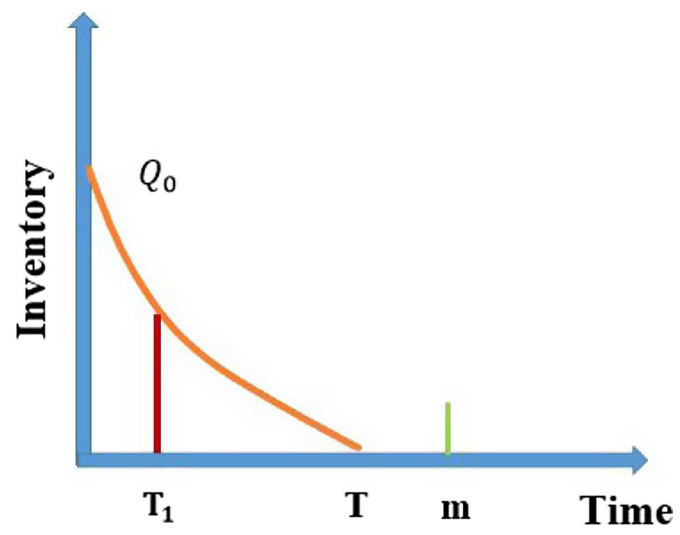

FIGURE 3. Inventory level versus time for Model III $m \geq T$.

Hence, the total earned during the cycle is

$$
\begin{aligned}
E_{3}= & p I_{e}\left\{-\alpha \beta\left(\frac{a T_{1}^{3}}{3 \theta}-\frac{T_{1}^{2}}{2 \theta}+\frac{b T_{1}^{2}}{2 \theta}\right)+\frac{x v^{y}}{2} T_{1}^{2}\left(\frac{1}{\theta}+1\right)\right. \\
& \left.+\frac{a \beta T_{1}^{2}}{3}+\frac{b \beta}{2} T_{1}^{2}\right\}+p I_{e}\left\{\beta\left(\frac{a T}{\theta}-\frac{1}{\theta}+\frac{b}{\theta}\right)-\frac{x v^{y}}{\theta}\right\}\left\{e^{\theta T}-\left(1+T_{1}\right) e^{\theta\left(T-T_{1}\right)}\right\} \\
& +p I_{e}\left(m-T_{1}\right)\left\{-\alpha \beta\left(\frac{a T_{1}^{2}}{3 \theta}-\frac{T_{1}}{2 \theta}+\frac{b T_{1}}{2 \theta}\right)+\frac{x v^{y}}{2} T_{1}\left(\frac{1}{\theta}+1\right)+\frac{a \beta T_{1}}{3}+\frac{b \beta}{2} T_{1}\right\}
\end{aligned}
$$




$$
+p I_{e}\left(m-T_{1}\right)\left\{\beta\left(\frac{a T}{\theta}-\frac{1}{\theta}+\frac{b}{\theta}\right)-\frac{x v^{y}}{\theta}\right\}\left\{e^{\theta T}-e^{\theta\left(T-T_{1}\right)}\right\} .
$$

There is no interest should be pay due to absence of unsold items. Therefore, the total average profit is

$$
\begin{aligned}
Z_{3}= & \frac{1}{T}\left[\left(P-c_{P}\right) \int_{0}^{T} D(I(t), t, v) \mathrm{d} t-c_{0}-v-c_{h} \int_{0}^{T} I(t) \mathrm{d} t-\theta c_{p} \int_{T_{1}}^{T} I(t) \mathrm{d} t+E_{3}\right] \\
= & \frac{1}{T}\left[\left(p-c_{p}\right)\left\{\beta\left(\frac{a T^{2}}{2}+b T\right)+x v^{y} T\right\}-\left(c_{0}+v\right)+p I_{e}\left\{-\alpha \beta\left(\frac{a T_{1}^{3}}{3 \theta}-\frac{T_{1}^{2}}{2 \theta^{2}}+\frac{b T_{1}^{2}}{2 \theta}\right)\right.\right. \\
& \left.+\frac{x v^{y}}{2} T_{1}^{2}\left(\frac{1}{\theta}+1\right)+\frac{a \beta T_{1}^{2}}{3}+\frac{b \beta}{2} T_{1}^{2}\right\}+p I_{e}\left\{\beta\left(\frac{a T}{\theta}-\frac{1}{\theta^{2}}+\frac{b}{\theta}\right)-\frac{x v^{y}}{\theta}\right\} \\
& \times\left\{e^{\theta T}-\left(1+T_{1}\right) e^{\theta\left(T-T_{1}\right)}\right\}+p I_{e}\left(m-T_{1}\right)\left\{-\alpha\left(\frac{a T_{1}^{2}}{3 \theta}-\frac{T_{1}}{2 \theta^{2}}+\frac{b T_{1}}{2 \theta}\right)\right. \\
& \left.+\frac{x v^{y}}{2} T_{1}\left(\frac{1}{\theta}+1\right)+\frac{a \beta T_{1}}{3}+\frac{b \beta}{2} T_{1}\right\}+p I_{e}\left(m-T_{1}\right)\left\{\beta\left(\frac{a T}{\theta}-\frac{1}{\theta^{2}}+\frac{b}{\theta}\right)\right. \\
& \left.-\frac{x v^{y}}{\theta}\right\}\left\{e^{\theta T}-e^{\theta\left(T-T_{1}\right)}\right\}+\left(p-c_{p}-c_{h}\right)(1+\alpha)\left\{-\beta\left(\frac{a T^{2}}{2 \theta}-\frac{T}{\theta^{2}}+\frac{b T}{\theta}\right)+\frac{x v^{y}}{\theta} T\right\} \\
& +\left(p-c_{p}-c_{h}\right)(1+\alpha)\left\{\beta\left(\frac{a T}{\theta}-\frac{1}{\theta^{2}}+\frac{b}{\theta}\right)-\frac{x v^{y}}{\theta}\right\} e^{\theta T}+\theta c_{p} \frac{a \beta}{2 \theta}\left(T^{2}-T_{1}^{2}\right) \\
& \left.-\theta c_{p}\left\{\beta\left(\frac{1}{\theta^{2}}-\frac{b}{\theta}\right)+\frac{x v^{y}}{\theta}\right\}\left(T-T_{1}\right)-\theta c_{p}\left\{\beta\left(\frac{a T}{\theta}-\frac{1}{\theta^{2}}+\frac{b}{\theta}\right)-\frac{x v^{y}}{\theta}\right\} e^{\theta\left(T-T_{1}\right)}\right] .
\end{aligned}
$$

$Z_{3}\left(T_{1}, T\right)$ is now maximized.

Lemma 3.4. The maximum value for $Z_{3}=\left(T_{1}, T\right)$ is obtain for the optimised values of $T$ and $T_{1}$, which satisfy the following equations

$$
\text { (i) } \begin{aligned}
p I_{e} & \left\{-\alpha \beta\left(\frac{a T_{1}^{2}}{\theta}-\frac{T_{1}}{\theta^{2}}+\frac{b T_{1}}{\theta}\right)+\frac{x v^{y}}{2} T_{1}\left(\frac{1}{\theta}+1\right)+a \beta T_{1}^{2}+b \beta T_{1}\right\} \\
& -p \theta I_{e}\left\{\beta\left(\frac{a T}{\theta}-\frac{1}{\theta^{2}}+\frac{b}{\theta}\right)-\frac{x v^{y}}{\theta}\right\}\left\{\left(1+T_{1}\right) e^{\theta\left(T-T_{1}\right)}\right\} \\
& -p I_{e}\left\{\beta\left(\frac{a T}{\theta}-\frac{1}{\theta^{2}}+\frac{b}{\theta}\right)-\frac{x v^{y}}{\theta}\right\} e^{\theta\left(T-T_{1}\right)}-p I_{e}\left\{-\alpha \beta\left(\frac{a T_{1}^{2}}{\theta}-\frac{T_{1}}{\theta^{2}}+\frac{b T_{1}}{\theta}\right)\right. \\
& \left.+\frac{x v^{y}}{\theta} T_{1}\right\}+p I_{e}\left(m-T_{1}\right)\left\{-\alpha \beta\left(\frac{a T_{1}}{\theta}-\frac{1}{\theta^{2}}+\frac{b}{\theta}\right)+\frac{x v^{y}}{\theta}\right\} \\
& -p I_{e}\left\{\beta\left(\frac{a T}{\theta}-\frac{1}{\theta^{2}}+\frac{b}{\theta}\right)-\frac{x v^{y}}{\theta}\right\}\left\{e^{\theta T}-e^{\theta\left(T-T_{1}\right)}\right\} \\
& -p \theta I_{e}\left\{\beta\left(\frac{a T}{\theta}-\frac{1}{\theta^{2}}+\frac{b}{\theta}\right)-\frac{x v^{y}}{\theta}\right\} e^{\theta\left(T-T_{1}\right)}-a \beta c_{p} T_{1}+\theta c_{p}\left\{\beta\left(\frac{1}{\theta^{2}}-\frac{b}{\theta}\right)\right. \\
& \left.+\frac{x v^{y}}{\theta}\right\}+\theta^{2} c_{p}\left\{\beta\left(\frac{a T}{\theta}-\frac{1}{\theta^{2}}+\frac{b}{\theta}\right)-\frac{x v^{y}}{\theta}\right\} e^{\theta\left(T-T_{1}\right)}=0 \\
(p & \left.-c_{p}\right)\left\{\beta(a T+b)+x v^{y}\right\}+p \theta I_{e}\left\{\beta\left(\frac{a T}{\theta}-\frac{1}{\theta^{2}}+\frac{b}{\theta}\right)-\frac{x v^{y}}{\theta}\right\} \\
& \times\left\{e^{\theta T}-\left(1-T_{1}\right) e^{\theta\left(T-T_{1}\right)}\right\}+\frac{a \beta p I_{e}}{\theta}\left\{e^{\theta T}-\left(1-T_{1}\right) e^{\theta\left(T-T_{1}\right)}\right\} \\
\text { (ii) } \quad &
\end{aligned}
$$




$$
\begin{aligned}
& +\left(m-T_{1}\right) p \theta I_{e}\left\{\beta\left(\frac{a T}{\theta}-\frac{1}{\theta^{2}}+\frac{b}{\theta}\right)-\frac{x v^{y}}{\theta}\right\}\left\{e^{\theta T}-\left(1-T_{1}\right) e^{\theta\left(T-T_{1}\right)}\right\} \\
& +\left(m-T_{1}\right) \frac{a \beta p I_{e}}{\theta}\left\{e^{\theta T}-\left(1-T_{1}\right) e^{\theta\left(T-T_{1}\right)}\right\}+\left(p-c_{p}-c_{h}\right)(1+\alpha) \\
& \times\left\{-\beta\left(\frac{a T}{\theta}-\frac{1}{\theta^{2}}+\frac{b}{\theta}\right)+\frac{x v^{y}}{\theta}\right\}+\theta\left(p-c_{p}-c_{h}\right)(1+\alpha)\left\{-\beta\left(\frac{a T}{\theta}\right.\right. \\
& \left.\left.-\frac{1}{\theta^{2}}+\frac{b}{\theta}\right)+\frac{x v^{y}}{\theta}\right\} e^{\theta T}+\left(p-c_{p}-c_{h}\right)(1+\alpha) \frac{a \beta}{\theta}+a \beta c_{p} T \\
& -\theta c_{p}\left\{\beta\left(\frac{1}{\theta^{2}}-\frac{b}{\theta}\right)+\frac{x v^{y}}{\theta}\right\}-\theta^{2} c_{p}\left\{\beta\left(\frac{a T}{\theta}-\frac{1}{\theta^{2}}+\frac{b}{\theta}\right)-\frac{x v^{y}}{\theta}\right\} e^{\theta\left(T-T_{1}\right)} \\
& -a \beta c_{p} e^{\theta\left(T-T_{1}\right)}=0
\end{aligned}
$$

provided the following sufficient conditions are satisfied

(i)

$$
\begin{aligned}
& p I_{e}\left\{-\alpha \beta\left(\frac{2 a T_{1}}{\theta}-\frac{1}{\theta^{2}}+\frac{b}{\theta}\right)+\frac{x v^{y}}{2}\left(\frac{1}{\theta}+1\right)+2 a \beta T_{1}+b \beta\right\} \\
& -p I_{e}\left\{\beta\left(\frac{a T}{\theta}-\frac{1}{\theta^{2}}+\frac{b}{\theta}\right)-\frac{x v^{y}}{\theta}\right\} e^{\theta\left(T-T_{1}\right)}+p \theta I_{e}\left\{\beta\left(\frac{a T}{\theta}-\frac{1}{\theta^{2}}+\frac{b}{\theta}\right)\right. \\
& \left.-\frac{x v^{y}}{\theta}\right\}\left(1+T_{1}\right) e^{\theta\left(T-T_{1}\right)}-2 p I_{e}\left\{-\alpha \beta\left(\frac{2 a T_{1}}{\theta}-\frac{1}{\theta^{2}}+\frac{b}{\theta}\right)+\frac{x v^{y}}{\theta}\right\} \\
& -\frac{a \alpha \beta p I_{e}}{\theta}\left(m-T_{1}\right)-p I_{e}\left(m-T_{1}\right)\left\{-\alpha \beta\left(\frac{a T_{1}}{\theta}-\frac{1}{\theta^{2}}+\frac{b}{\theta}\right)\right. \\
& \left.+\frac{x v^{y}}{\theta}\right\}-p \theta I_{e}\left\{\beta\left(\frac{a T}{\theta}-\frac{1}{\theta^{2}}+\frac{b}{\theta}\right)-\frac{x v^{y}}{\theta}\right\} e^{\theta\left(T-T_{1}\right)} \\
& -p I_{e}\left\{\beta\left(\frac{a T}{\theta}-\frac{1}{\theta^{2}}+\frac{b}{\theta}\right)-\frac{x v^{y}}{\theta}\right\} e^{\theta\left(T-T_{1}\right)}-p \theta^{2} I_{e}\left(m-T_{1}\right)\left\{\beta\left(\frac{a T}{\theta}-\frac{1}{\theta^{2}}+\frac{b}{\theta}\right)\right. \\
& \left.-\frac{x v^{y}}{\theta}\right\} e^{\theta\left(T-T_{1}\right)} a \beta c_{p}-\theta^{3} c_{p}\left\{\beta\left(\frac{a T}{\theta}-\frac{1}{\theta^{2}}+\frac{b}{\theta}\right)-\frac{x v^{y}}{\theta}\right\} e^{\theta\left(T-T_{1}\right)}=0 \\
& \left(p-c_{p}\right) a \beta+p \theta^{2} I_{e}\left\{\beta\left(\frac{a T}{\theta}-\frac{1}{\theta^{2}}+\frac{b}{\theta}\right)-\frac{x v^{y}}{\theta}\right\}\left\{e^{\theta T}-\left(1+T_{1}\right) e^{\theta\left(T-T_{1}\right)}\right\} \\
& +\frac{a \beta p I_{e}}{\theta}\left\{e^{\theta T}-\left(1+T_{1}\right) e^{\theta\left(T-T_{1}\right)}\right\}+\left(m-T_{1}\right) p \theta^{2} I_{e}\left\{\beta\left(\frac{a T}{\theta}-\frac{1}{\theta^{2}}+\frac{b}{\theta}\right)\right. \\
& \left.-\frac{x v^{y}}{\theta}\right\}\left\{e^{\theta T}-\left(1+T_{1}\right) e^{\theta\left(T-T_{1}\right)}\right\}+2\left(m-T_{1}\right) a \beta p I_{e}\left\{e^{\theta T}-e^{\theta\left(T-T_{1}\right)}\right\} \\
& +\theta^{2}\left(p-c_{p}-c_{h}\right)(1+\alpha)\left\{\beta\left(\frac{a T}{\theta}-\frac{1}{\theta^{2}}+\frac{b}{\theta}\right)+\frac{x v^{y}}{\theta}\right\} e^{\theta T} \\
& +\theta\left(p-c_{p}-c_{h}\right)(1+\alpha) \frac{a \beta}{\theta} e^{\theta T}+\theta\left(p-c_{p}-c_{h}\right)(1+\alpha) a \beta e^{\theta T}+a \beta c_{p} \\
& >\theta\left(p-c_{p}-c_{h}\right)(1+\alpha) \frac{a \beta}{\theta}+\theta^{3} c_{p}\left\{\beta\left(\frac{a T}{\theta}-\frac{1}{\theta^{2}}+\frac{b}{\theta}\right)-\frac{x v^{y}}{\theta}\right\} e^{\theta\left(T-T_{1}\right)} \\
& -2 a \beta \theta c_{p} e^{\theta\left(T-T_{1}\right)} \\
& \left(1+m+\theta c_{P}\right) p I_{e}\left\{\beta\left(\frac{a T}{\theta}-\frac{1}{\theta^{2}}+\frac{b}{\theta}\right)-\frac{x v^{y}}{\theta}\right\} e^{\theta\left(T-T_{1}\right)} \\
& +\left(\theta c_{p}-1\right) \frac{a \beta p I_{e}}{\theta} e^{\theta\left(T-T_{1}\right)}>p I_{e}\left\{\beta\left(\frac{a T}{\theta}-\frac{1}{\theta^{2}}+\frac{b}{\theta}\right)-\frac{x v^{y}}{\theta}\right\} e^{\theta T} .
\end{aligned}
$$$$
\text { (ii) }
$$ 
[See Appendix D for proof].

One has to find maximum value of

$$
Z\left(T_{1}, T\right)=\left\{\begin{array}{ll}
Z_{1}\left(T_{1}, T\right) & m \leq T_{1}<T \\
Z_{2}\left(T_{1}, T\right) & T_{1}<m<T \\
Z_{3}\left(T_{1}, T\right) & m \geq T
\end{array}\right\}
$$

\section{NUMERICAL EXAMPLES}

To prove the applicability of this model a numerical example is discussed here for the three cases (which are discussed in the Model formulation section). For the numerical result, the parametric values for inventory system are considered as on Khanra et al. [19], which are as follows: $a=200$ units per month, $b=1.8$ units per month, $\alpha=0.002$ units per month, $v=\$ 2$ per unit, $\beta=0.6$ unit per month, $x=0.7$ unit per month, $y=0.7$ unit per month, $c_{0}=\$ 90$ per order, $c_{p}=\$ 1.8$ per order, $I_{r}=\$ 0.12$ per month, $I_{e}=\$ 0.10$ per month, $p=\$ 7$ per unit, $c_{h}=\$ 1.2$ per month, $p=\$ 2$ per unit and $m=0.3$ year. Then by using the equations (3.3), (3.5), and (3.7), the optimum results for different cases are shown in the following Table 2. From the optimum result Table 2, one can easily find that the total profit is $\$ 1488.85$ when permissible delay period is less then length of time in which inventory reach the zero level and profit is $\$ 1591.56$, when delay period is grater than total cycle time but the optimum profit is obtain when delay period is in between cycle time and time length when inventory reach to the zero level. The bold values shows the optimal result of this model.

TABLE 2. Optimal result for different cases.

\begin{tabular}{llll}
\hline \hline & $\begin{array}{l}\text { Cycle Length } \\
T^{*} \text { (Year) }\end{array}$ & $\begin{array}{l}\text { Time length } \\
\text { inventory reach } \\
\text { zero level } T_{1}^{*}(\text { Year) }\end{array}$ & $\begin{array}{l}\text { Total profit } \\
Z_{i}^{*}\left(T^{*}, T_{1}^{*}\right)(\$)\end{array}$ \\
\hline $\begin{array}{l}\text { Case I: } \\
m \leq T_{1} \leq T\end{array}$ & 1.65 & 0.42 & $\$ 1488.85$ \\
$\begin{array}{l}\text { Case II: } \\
T_{1}<m<T\end{array}$ & $\mathbf{0 . 9 2}$ & $\mathbf{0 . 5 3}$ & $\mathbf{\$ 1 8 4 0 . 4 1}$ \\
$\begin{array}{l}\text { Case III: } \\
m \geq T\end{array}$ & 1.13 & 0.74 & $\$ 1591.56$ \\
\hline
\end{tabular}

Hence, the maximum average profit in this case is $Z_{2}^{*}\left(T_{1}, T\right)=\$ 1840.41$ where $T_{1}^{*}=0.53$ year, $T^{*}=0.92$ year.

\section{Sensitivity ANALYSiS}

In this section contained the effect of change of the parametric values on total profit by increasing and decreasing $50 \%$ and $20 \%$ of the parameter $a, b, \alpha, \beta, x, y, c_{p}, v, I_{e}, h$, and $C_{0}$ respectively.

The effect of change for total profit is shown in Table 3 and a graphical representation (Fig. 4) also provide to show the effect of parametric value over the total profit:

From the sensitivity analysis Table 3, one can easily find that the effect of different parameters as follows:

(i) The effect of scaling parameter $a$ is very much sensitive, small change in $a$, there is a huge impact on total system profit.

(ii) The parameter $\beta$ is much more effective in total system profit. Increase or decrease in $\beta$ is very sensitive for total profit.

(iii) The production cost $c_{p}$ has a great impact in the total system profit.

(iv) The holding cost is more sensitive as usual. Increase of holding cost is always harmful for any production industry. 
(v) The initial setup cost is also very crucial factor for any industry, that was clearly shown in the sensitivity analysis table.

(vi) The earned interest $I_{e}$ is quite sensitive for any economic order quantity model that was clear from the sensitivity analysis table.

(vii) The shape parameter $y$ for advertising parameter is little bit sensitive in the calculation of profit of the system.

(viii) The other parameters $b, \alpha, v$, and $x$ are little bit sensitive in the profit calculation.

TABLE 3. Sensitivity analysis table.

\begin{tabular}{|c|c|c|c|c|c|c|c|}
\hline Parameters & $\begin{array}{l}\text { Change(in \%) } \\
\text { (in \%) }\end{array}$ & $\begin{array}{l}\text { Optimal } \\
\text { profit } \\
Z\left(T_{1}^{*}, T^{*}\right) \\
\end{array}$ & $\begin{array}{l}\text { Change } \\
Z\left(T_{1}^{*}, T^{*}\right)\end{array}$ & Parameters & $\begin{array}{l}\text { Change } \\
\text { (in \%) }\end{array}$ & $\begin{array}{l}\text { Optimal } \\
\text { profit } \\
Z\left(T_{1}^{*}, T^{*}\right) \\
\end{array}$ & $\begin{array}{l}\text { Change } \\
Z\left(T_{1}^{*}, T^{*}\right)\end{array}$ \\
\hline \multirow{4}{*}{$a$} & $50 \%$ & 2809.15 & +52.64 & \multirow{4}{*}{$\beta$} & $50 \%$ & 2808.64 & +52.61 \\
\hline & $25 \%$ & 2324.78 & +26.32 & & $25 \%$ & 2324.52 & +26.30 \\
\hline & $-25 \%$ & 1356.03 & -26.32 & & $-25 \%$ & 1356.29 & -26.30 \\
\hline & $-50 \%$ & 871.66 & -52.64 & & $-50 \%$ & 872.17 & -52.61 \\
\hline \multirow{4}{*}{$b$} & $50 \%$ & 1844.50 & +0.22 & \multirow{4}{*}{$x$} & $50 \%$ & 1842.37 & +00.11 \\
\hline & $25 \%$ & 1842.45 & +00.11 & & $25 \%$ & 1841.39 & +0.05 \\
\hline & $-25 \%$ & 1838.36 & -00.11 & & $-25 \%$ & 1839.42 & -0.05 \\
\hline & $-50 \%$ & 1836.32 & -00.22 & & $-50 \%$ & 1838.44 & -00.11 \\
\hline \multirow{4}{*}{$\alpha$} & $50 \%$ & 1842.56 & +0.12 & \multirow{4}{*}{$y$} & $50 \%$ & 1841.48 & +0.059 \\
\hline & $25 \%$ & 1841.49 & +0.06 & & $25 \%$ & 1840.91 & +0.028 \\
\hline & $-25 \%$ & 1839.33 & -0.06 & & $-25 \%$ & 1839.96 & -0.024 \\
\hline & $-50 \%$ & 1838.25 & -0.12 & & $-50 \%$ & 1839.56 & -0.046 \\
\hline \multirow{4}{*}{$v$} & $50 \%$ & 1840.61 & +0.011 & \multirow{4}{*}{$c_{p}$} & $50 \%$ & 1498.97 & -18.55 \\
\hline & $25 \%$ & 1840.53 & +0.006 & & $25 \%$ & 1513.50 & -17.76 \\
\hline & $-25 \%$ & 1840.23 & -0.009 & & $-25 \%$ & 2167.31 & +17.76 \\
\hline & $-50 \%$ & 1839.98 & -0.023 & & $-50 \%$ & 2494.22 & $\begin{array}{r}+35.52 \\
\end{array}$ \\
\hline \multirow{4}{*}{$I_{e}$} & $50 \%$ & 1683.64 & -8.52 & \multirow{4}{*}{$h$} & $50 \%$ & 1513.55 & -17.76 \\
\hline & $25 \%$ & 1762.02 & -4.26 & & $25 \%$ & 1676.98 & -8.88 \\
\hline & $-25 \%$ & 1918.79 & +4.26 & & $-25 \%$ & 2003.84 & +8.88 \\
\hline & $-50 \%$ & 1997.17 & $\begin{array}{l}+8.52 \\
\end{array}$ & & $-50 \%$ & 2167.26 & $\begin{array}{l}+17.76 \\
\end{array}$ \\
\hline \multirow{4}{*}{$C_{0}$} & $50 \%$ & 1791.49 & -2.66 & & & & \\
\hline & $25 \%$ & 1815.95 & -1.33 & & & & \\
\hline & $-20 \%$ & 1864.86 & +1.33 & & & & \\
\hline & $-50 \%$ & 1889.32 & $\begin{array}{r}+2.66 \\
+\end{array}$ & & & & \\
\hline
\end{tabular}

\section{MANAGerial insights}

This model deals with an inventory model for items with stock level and advertising dependent demand pattern by considering shortages under trade-credit policy. The managerial insights of this chapter are given as follows.

(i) The main aim of any inventory model to optimise profit. DSL has a great impact on optimization of profit. The vendor always want to sell his own product more, compare to other product. The common policy for any vendor that no customer will go away from his/her shop with out the required product, and can easily see the stock of product of his/her shop. Normally the demand will increase if the stored product is higher than the demand. Casually, profit will optimised if vendor can sell more product. In this model one can easily find out that, when demand depend on the on-hand inventory, the profit was optimised. 


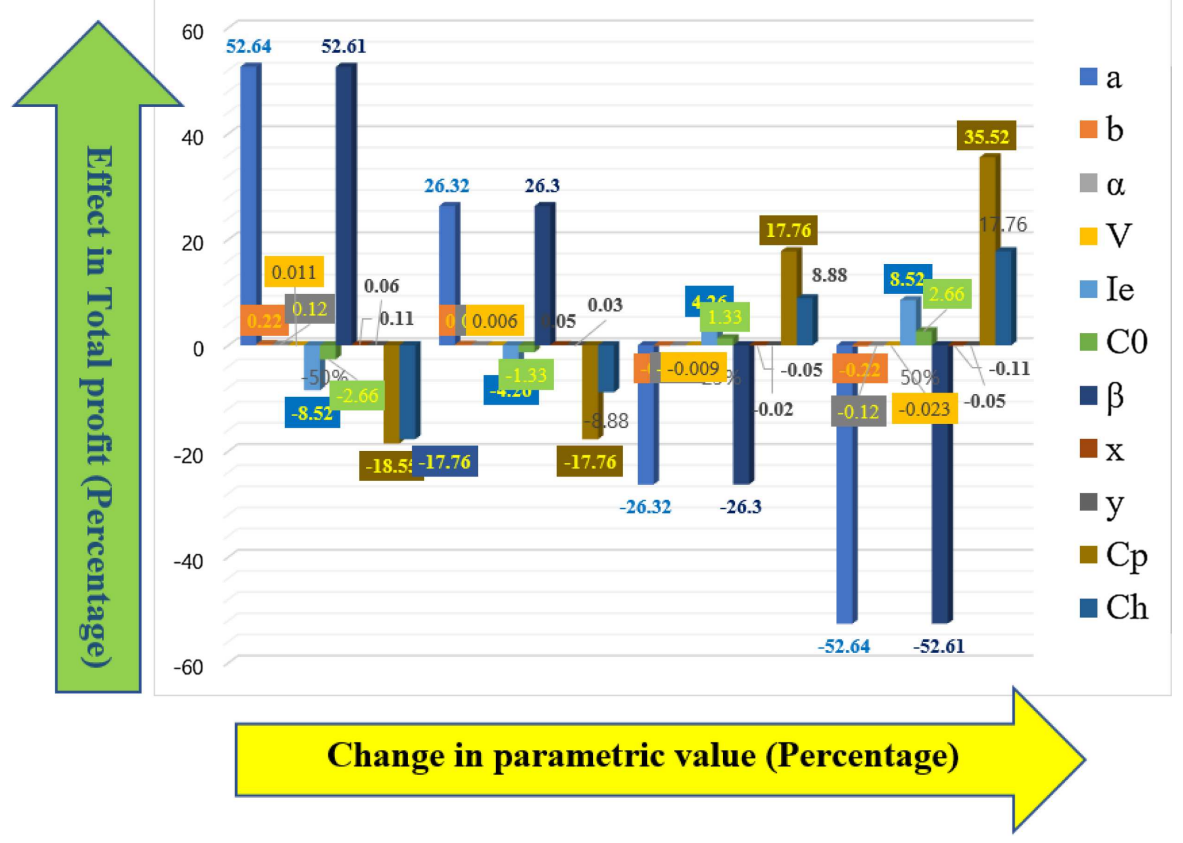

FIGURE 4. Effect of change in parametric value in total profit.

(ii) Any company wants to sell more items by using different type of sales advertisement policy. The main aim of advertising is to aware or inform the general public about the product, it can compared the difference of the other products and influence the common public to buy a particular product. Thus the sell of a particular product's automatically increase by a better advertisement.

Thus advertising for any product is one of the most crucial factor in any industry to increase the profit in modern competitive marketing environment.

(iii) To increase the sell, retailer/supplier offer a certain credit period to their customers without any penalty during the said delay time period, it motivates customer to order more quantities. In this regard the trade credit policy is considered in this model. Thus, sell of a particular product increases by considering tradecredit policy, which directly optimize the total system profit. Thus, from the managerial point of view, this strategy is valid and useful to this competitive business market.

\section{Conclusions}

The proposed model extends the existing literature by considering finite replenishment rate, stock-dependent and advertising demand and two progressive periods for delay-in-payments. The analytic result proves the global optimality of the solutions. Some theorems also proved to show that the optimal solution is global. The numerical results proved that the model obtained the global optimum profit at the optimal solutions. This model can be extended by considering shortages of the product, deterioration which is time-varying, for multi-item perishable products (see [33]). This model can also be extended by considering environmental effect or by considering the effect of carbon emission $[1,25,41]$, which is one of the most important issue in today's business environment. One can develop this model to a supply chain model along with the consideration of variable production rate and variable setup cost [9,34]. To identify the deteriorate items one can use the autonomation policy [10] along with the latest Radio Frequency Identify Device (RFID) technology [55]. This will be another good research if one considered energy consumption for carbon emission [2] along with increased system reliability [16]. The fully deteriorated items are treated as waste, those waste items management is one another interesting research in this direction $[42,45,53]$. 


\section{Appendix A.}

Proof. One can have $U\left(T_{1}, T\right)=\frac{1}{T} W\left(T_{1}, T\right)$. For maximum value of $U\left(T_{1}, T\right)$ at $T_{1}=T_{1}^{*}, T=T^{*}$, necessary condition are $\frac{\partial U\left(T_{1}, T\right)}{\partial T_{1}}=0$ and $\frac{\partial U\left(T_{1}, T\right)}{\partial T}=0$.

Now

$$
\frac{\partial U\left(T_{1}, T\right)}{\partial T}=\frac{T \frac{\partial W\left(T_{1}, T\right)}{\partial T}-W\left(T_{1}, T\right)}{T^{2}} \text { and } \quad \frac{\partial U\left(T_{1}, T\right)}{\partial T_{1}}=\frac{1}{T} \frac{\partial W\left(T_{1}, T\right)}{\partial T_{1}}
$$

Therefore, $\frac{\partial U\left(T_{1}, T\right)}{\partial T}=0$ gives $T \frac{\partial W\left(T_{1}, T\right)}{\partial T}-W\left(T_{1}, T\right)=0$ and $\frac{\partial U\left(T_{1}, T\right)}{\partial T_{1}}=0$ gives $\frac{\partial W\left(T_{1}, T\right)}{\partial T_{1}}=0$. This equations are satisfied if one considered $T=T^{*}$ and $T_{1}=T_{1}^{*}$. Again,

$$
\frac{\partial^{2} U\left(T_{1}, T\right)}{\partial T^{2}}=\frac{T\left(T \frac{\partial^{2} W\left(T_{1}, T\right)}{\partial T^{2}}+\frac{\partial W\left(T_{1}, T\right)}{\partial T}-\frac{\partial W\left(T_{1}, T\right)}{\partial T}\right)-2 T\left(T \frac{\partial W\left(T_{1}, T\right)}{\partial T}-W\left(T_{1}, T\right)\right)}{T^{4}} .
$$

Thus, for $T=T^{*}$ and $T_{1}=T_{1}^{*}$, one can write $\frac{\partial^{2} U\left(T_{1}, T\right)}{\partial T^{2}}=\frac{1}{T} \frac{\partial^{2} W\left(T_{1}, T\right)}{\partial T^{2}}$.

Similarly, at $T=T^{*}$ and $T_{1}=T_{1}^{*}$, one can obtain $\frac{\partial^{2} U\left(T_{1}, T\right)}{\partial T_{1}^{2}}=\frac{1}{T} \frac{\partial^{2} W\left(T_{1}, T\right)}{\partial T_{1}^{2}}$ and $\frac{\partial^{2} U\left(T_{1}, T\right)}{\partial T \partial T_{1}}=\frac{1}{T} \frac{\partial^{2} W\left(T_{1}, T\right)}{\partial T \partial T_{1}}$. Hence,

$$
\left|\begin{array}{ll}
\frac{\partial^{2} U\left(T_{1}, T\right)}{\partial T_{1}^{2}} & \frac{\partial^{2} U\left(T_{1}, T\right)}{\partial T_{1} \partial T} \\
\frac{\partial^{2} U\left(T_{1}, T\right)}{\partial T_{1} \partial T} & \frac{\partial^{2} U\left(T_{1}, T\right)}{\partial T^{2}}
\end{array}\right|=\left|\begin{array}{cc}
\frac{1}{T} \frac{\partial^{2} W\left(T_{1}, T\right)}{\partial T_{1}^{2}} & \frac{1}{T} \frac{\partial^{2} W\left(T_{1}, T\right)}{\partial T_{1} \partial T} \\
\frac{1}{T} \frac{\partial^{2} W\left(T_{1}, T\right)}{\partial T_{1} \partial T} & \frac{1}{T} \frac{\partial^{2} W\left(T_{1}, T\right)}{\partial T^{2}}
\end{array}\right|=\frac{1}{T^{2}}\left|\begin{array}{cc}
\frac{\partial^{2} W\left(T_{1}, T\right)}{\partial T_{1}^{2}} & \frac{\partial^{2} W\left(T_{1}, T\right)}{\partial T_{1} \partial T} \\
\frac{\partial^{2} W\left(T_{1}, T\right)}{\partial T_{1} \partial T} & \frac{\partial^{2} W\left(T_{1}, T\right)}{\partial T^{2}}
\end{array}\right| .
$$

Thus,

$$
\begin{gathered}
\text { if }\left|\begin{array}{ll}
\frac{\partial^{2} W\left(T_{1}, T\right)}{\partial T_{1}^{2}} & \frac{\partial^{2} W\left(T_{1}, T\right)}{\partial T_{1} \partial T} \\
\frac{\partial^{2} W\left(T_{1}, T\right)}{\partial T_{1} \partial T} & \frac{\partial^{2} W\left(T_{1}, T\right)}{\partial T^{2}}
\end{array}\right|>0 \quad \text { and } \quad \frac{\partial^{2} W\left(T_{1}, T\right)}{\partial T_{1}^{2}}<0 \\
\text { then }\left|\begin{array}{ll}
\frac{\partial^{2} U\left(T_{1}, T\right)}{\partial T_{1}^{2}} & \frac{\partial^{2} U\left(T_{1}, T\right)}{\partial T_{1} \partial T} \\
\frac{\partial^{2} U\left(T_{1}, T\right)}{\partial T_{1} \partial T} & \frac{\partial^{2} U\left(T_{1}, T\right)}{\partial T^{2}}
\end{array}\right|>0 \quad \text { and } \quad \frac{\partial^{2} U\left(T_{1}, T\right)}{\partial T_{1}^{2}}<0
\end{gathered}
$$

which indicates $U\left(T_{1}, T\right)$ is maximum.

\section{Appendix B.}

Proof. To maximize $Z_{1}\left(T_{1}, T\right)=\frac{1}{T} W_{1}\left(T_{1}, T\right)$, the necessary conditions are as $\frac{\partial Z_{1}\left(T_{1}, T\right)}{\partial T_{1}}=0, \frac{\partial Z_{1}\left(T_{1}, T\right)}{\partial T}=0$ and the above theorem indicate the sufficiency of the condition are

$$
\frac{\partial^{2} W_{1}\left(T_{1}, T\right)}{\partial^{2} T_{1}}<0, \quad \text { and } \quad\left|\begin{array}{ll}
\frac{\partial^{2} W_{1}\left(T_{1}, T\right)}{\partial T_{1}^{2}} & \frac{\partial^{2} W_{1}\left(T_{1}, T\right)}{\partial T_{1} \partial T} \\
\frac{\partial^{2} W_{1}\left(T_{1}, T\right)}{\partial T_{1} \partial T} & \frac{\partial^{2} W_{1}\left(T_{1}, T\right)}{\partial T^{2}}
\end{array}\right|>0
$$

Now, $\frac{\partial Z_{1}\left(T_{1}, T\right)}{\partial T_{1}}=0$ gives

$$
\begin{aligned}
p I_{r} & \left\{\frac{\alpha \beta}{\theta} T_{1}-\beta\left(\frac{1}{\theta^{2}}-\frac{b}{\theta}-\frac{x v^{y}}{\theta}\right)\right\}-\left\{\beta\left(\frac{a T}{\theta}-\frac{1}{\theta^{2}}+\frac{b}{\theta}\right)-\frac{x v^{y}}{\theta}\right\} e^{\theta\left(T-T_{1}\right)} \\
& -\theta c_{p}\left\{\frac{\alpha \beta}{\theta} T_{1}-\beta\left(\frac{1}{\theta^{2}}-\frac{b}{\theta}\right)-\frac{x v^{y}}{\theta}\right\}+\theta c_{p}\left\{\beta\left(\frac{a T}{\theta}-\frac{1}{\theta^{2}}+\frac{b}{\theta}\right)-\frac{x v^{y}}{\theta}\right\} e^{\theta\left(T-T_{1}\right)} \\
& =0 .
\end{aligned}
$$


Also, $\frac{\partial Z_{1}\left(T_{1}, T\right)}{\partial T}=0$ provides

(ii)

$$
\begin{aligned}
(p- & \left.c_{p}\right)\left\{\beta(a T+b)+\frac{x v^{y}}{\theta}\right\}+p I_{e}\left\{\beta\left(\frac{a T}{\theta}-\frac{1}{\theta}+\frac{b}{\theta}\right)-\frac{x v^{y}}{\theta}\right\}\left\{e^{\theta T}-(1+m) e^{\theta(T-m)}\right\} \\
& +\frac{p I_{e} \alpha \beta}{\theta}\left\{e^{\theta T}-(1+m) e^{\theta(T-m)}\right\}+\left(p-c_{p}-c_{h}\right)(1+\alpha)\left\{-\beta\left(\frac{a T}{\theta}-\frac{1}{\theta^{2}}+\frac{b}{\theta}\right)+\frac{x v^{y}}{\theta}\right\} \\
& +\left(p-c_{p}-c_{h}\right)(1+\alpha)\left\{\beta\left(\frac{a T}{\theta}-\frac{1}{\theta^{2}}+\frac{b}{\theta}\right)-\frac{x v^{y}}{\theta}\right\} e^{\theta T}+\left(p-c_{p}-c_{h}\right)(1+\alpha) \frac{a \beta}{\theta} e^{\theta T} \\
& -p \theta I_{r}\left\{\beta\left(\frac{a T}{\theta}-\frac{1}{\theta^{2}}+\frac{b}{\theta}\right)-\frac{x v^{y}}{\theta}\right\}\left\{e^{\theta(T-m)}-e^{\theta\left(T-T_{1}\right)}\right\}-p \alpha \beta I_{r}\left\{e^{\theta(T-m)}-e^{\theta\left(T-T_{1}\right)}\right\} \\
& +\alpha \beta c_{p} T-\theta c_{p}\left\{\beta\left(\frac{1}{\theta^{2}}-\frac{b}{\theta}\right)+\frac{x v^{y}}{\theta}\right\}+a \beta c_{p} e^{\theta\left(T-T_{1}\right)}+\theta^{2} c_{p}\left\{\beta\left(\frac{a T}{\theta}-\frac{1}{\theta^{2}}+\frac{b}{\theta}\right)-\frac{x v^{y}}{\theta}\right\} e^{\theta\left(T-T_{1}\right)} \\
= & 0 .
\end{aligned}
$$

By solving the equations, one can obtain optimal values of $T_{1}$ and $T$. Using the above theorem,

$$
\frac{\partial^{2} W_{1}\left(T_{1}, T\right)}{\partial^{2} T_{1}}<0, \quad \text { and } \quad\left|\begin{array}{ll}
\frac{\partial^{2} W_{1}\left(T_{1}, T\right)}{\partial T_{1}^{2}} & \frac{\partial^{2} W_{1}\left(T_{1}, T\right)}{\partial T_{1} \partial T} \\
\frac{\partial^{2} W_{1}\left(T_{1}, T\right)}{\partial T_{1} \partial T} & \frac{\partial^{2} W_{1}\left(T_{1}, T\right)}{\partial T^{2}}
\end{array}\right|>0 .
$$

The following results are obtained

$$
\text { (i) } \begin{aligned}
& \frac{p I_{r} \alpha \beta}{\theta}-p I_{r}\left\{\beta\left(\frac{a T}{\theta}-\frac{1}{\theta^{2}}+\frac{b}{\theta}\right)-\frac{x v^{y}}{\theta}\right\} e^{\theta\left(T-T_{1}\right)} \\
& >\alpha \beta c_{p}-p I_{r} \theta c_{p}\left\{\beta\left(\frac{a T}{\theta}-\frac{1}{\theta^{2}}+\frac{b}{\theta}\right)-\frac{x v^{y}}{\theta}\right\} e^{\theta\left(T-T_{1}\right)}
\end{aligned}
$$

and

$$
\begin{aligned}
(p & \left.-c_{p}\right) \alpha \beta+p I_{e} \theta\left\{\beta\left(\frac{a T}{\theta}-\frac{1}{\theta^{2}}+\frac{b}{\theta}\right)-\frac{x v^{y}}{\theta}\right\}\left\{e^{\theta T}-(1+m) e^{\theta(T-m)}\right\} \\
& +\left(p-c_{p}-c_{h}\right)(1+\alpha)\left(\frac{1+\theta}{\theta}\right) a \beta e^{\theta T} \\
& +\theta\left(p-c_{p}-c_{h}\right)(1+\alpha)\left\{\beta\left(\frac{a T}{\theta}-\frac{1}{\theta^{2}}+\frac{b}{\theta}\right)-\frac{x v^{y}}{\theta}\right\} e^{\theta T}+a \beta c_{p} \\
& >\left(p-c_{p}-c_{h}\right)(1+\alpha) \frac{\alpha \beta}{\theta}+p a \beta I_{r} \frac{1+\theta}{\theta}\left\{e^{\theta(T-m)}-e^{\theta\left(T-T_{1}\right)}\right\} \\
& +p \theta^{2} I_{r}\left\{\beta\left(\frac{a T}{\theta}-\frac{1}{\theta^{2}}+\frac{b}{\theta}\right)-\frac{x v^{y}}{\theta}\right\}\left\{e^{\theta(T-m)}-e^{\theta\left(T-T_{1}\right)}\right\}
\end{aligned}
$$

(iii)

$$
\begin{aligned}
\theta c_{p} & \left\{\beta\left(\frac{a T}{\theta}-\frac{1}{\theta^{2}}+\frac{b}{\theta}\right)-\frac{x v^{y}}{\theta}\right\} e^{\theta\left(T-T_{1}\right)}+a \beta c_{p} e^{\theta\left(T-T_{1}\right)} \\
& >p I_{r} \theta\left\{\beta\left(\frac{a T}{\theta}-\frac{1}{\theta^{2}}+\frac{b}{\theta}\right)-\frac{x v^{y}}{\theta}\right\} e^{\theta\left(T-T_{1}\right)}+\frac{a \beta p I_{r}}{\theta} e^{\theta\left(T-T_{1}\right)} .
\end{aligned}
$$




\section{Appendix C.}

Proof. To maximize $Z_{2}\left(T_{1}, T\right)=\frac{1}{T} W_{2}\left(T_{1}, T\right)$, the necessary conditions are as $\frac{\partial Z_{2}\left(T_{1}, T\right)}{\partial T_{1}}=0, \frac{\partial Z_{2}\left(T_{1}, T\right)}{\partial T}=0$ and the sufficient condition are $\frac{\partial^{2} W_{2}\left(T_{1}, T\right)}{\partial^{2} T_{1}}<0$, and

$$
\left|\begin{array}{ll}
\frac{\partial^{2} W_{2}\left(T_{1}, T\right)}{\partial T_{1}^{2}} & \frac{\partial^{2} W_{2}\left(T_{1}, T\right)}{\partial T_{1} \partial T} \\
\frac{\partial^{2} W_{2}\left(T_{1}, T\right)}{\partial T_{1} \partial T} & \frac{\partial^{2} W_{2}\left(T_{1}, T\right)}{\partial T^{2}}
\end{array}\right|>0 .
$$

Now, $\frac{\partial Z_{2}\left(T_{1}, T\right)}{\partial T_{1}}=0$ gives

$$
\begin{aligned}
p I_{e} & \left\{-\alpha \beta\left(\frac{a T_{1}^{2}}{\theta}-\frac{T_{1}}{\theta^{2}}+\frac{b T_{1}}{\theta}\right)+\frac{x v^{y}}{2} T_{1}\left(\frac{1}{\theta}+1\right)+a \beta T_{1}^{2}+b \beta T_{1}\right\} \\
& -p \theta I_{e}\left\{\beta\left(\frac{a T}{\theta}-\frac{1}{\theta^{2}}+\frac{b}{\theta}\right)-\frac{x v^{y}}{\theta}\right\}\left\{\left(1+T_{1}\right) e^{\theta\left(T-T_{1}\right)}\right\} \\
& -p I_{e}\left\{\beta\left(\frac{a T}{\theta}-\frac{1}{\theta^{2}}+\frac{b}{\theta}\right)-\frac{x v^{y}}{\theta}\right\} e^{\theta\left(T-T_{1}\right)} \\
& -p I_{e}\left\{-\alpha \beta\left(\frac{a T_{1}^{2}}{\theta}-\frac{T_{1}}{\theta^{2}}+\frac{b T_{1}}{\theta}\right)+\frac{x v^{y}}{\theta} T_{1}\right\} \\
& +p I_{e}\left(m-T_{1}\right)\left\{-\alpha \beta\left(\frac{a T_{1}}{\theta}-\frac{1}{\theta^{2}}+\frac{b}{\theta}\right)+\frac{x v^{y}}{\theta}\right\} \\
& -p I_{e}\left\{\beta\left(\frac{a T}{\theta}-\frac{1}{\theta^{2}}+\frac{b}{\theta}\right)-\frac{x v^{y}}{\theta}\right\}\left\{e^{\theta T}-e^{\theta\left(T-T_{1}\right)}\right\} \\
& -p \theta I_{e}\left\{\beta\left(\frac{a T}{\theta}-\frac{1}{\theta^{2}}+\frac{b}{\theta}\right)-\frac{x v^{y}}{\theta}\right\} e^{\theta\left(T-T_{1}\right)}-a \beta c_{p} T_{1}+\theta c_{p}\left\{\beta\left(\frac{1}{\theta^{2}}-\frac{b}{\theta}\right)+\frac{x v^{y}}{\theta}\right\} \\
+ & \theta^{2} c_{p}\left\{\beta\left(\frac{a T}{\theta}-\frac{1}{\theta^{2}}+\frac{b}{\theta}\right)-\frac{x v^{y}}{\theta}\right\} e^{\theta\left(T-T_{1}\right)}=0 .
\end{aligned}
$$

Also, $\frac{\partial Z_{2}\left(T_{1}, T\right)}{\partial T}=0$ provides

$$
\begin{aligned}
(p & \left.-c_{p}\right)\left\{\beta(a T+b)+x v^{y}\right\}+p \theta I_{e}\left\{\beta\left(\frac{a T}{\theta}-\frac{1}{\theta^{2}}+\frac{b}{\theta}\right)\right. \\
& \left.-\frac{x v^{y}}{\theta}\right\}\left\{e^{\theta T}-\left(1-T_{1}\right) e^{\theta\left(T-T_{1}\right)}\right\}+\frac{a \beta p I_{e}}{\theta}\left\{e^{\theta T}-\left(1-T_{1}\right) e^{\theta\left(T-T_{1}\right)}\right\} \\
& +\left(m-T_{1}\right) p \theta I_{e}\left\{\beta\left(\frac{a T}{\theta}-\frac{1}{\theta^{2}}+\frac{b}{\theta}\right)-\frac{x v^{y}}{\theta}\right\}\left\{e^{\theta T}-\left(1-T_{1}\right) e^{\theta\left(T-T_{1}\right)}\right\} \\
& +\left(m-T_{1}\right) \frac{a \beta p I_{e}}{\theta}\left\{e^{\theta T}-\left(1-T_{1}\right) e^{\theta\left(T-T_{1}\right)}\right\} \\
& +\left(p-c_{p}-c_{h}\right)(1+\alpha)\left\{-\beta\left(\frac{a T}{\theta}-\frac{1}{\theta^{2}}+\frac{b}{\theta}\right)+\frac{x v^{y}}{\theta}\right\} \\
& +\theta\left(p-c_{p}-c_{h}\right)(1+\alpha)\left\{-\beta\left(\frac{a T}{\theta}-\frac{1}{\theta^{2}}+\frac{b}{\theta}\right)+\frac{x v^{y}}{\theta}\right\} e^{\theta T} \\
& +\left(p-c_{p}-c_{h}\right)(1+\alpha) \frac{a \beta}{\theta}+a \beta c_{p} T-\theta c_{p}\left\{\beta\left(\frac{1}{\theta^{2}}-\frac{b}{\theta}\right)+\frac{x v^{y}}{\theta}\right\} \\
& -\theta^{2} c_{p}\left\{\beta\left(\frac{a T}{\theta}-\frac{1}{\theta^{2}}+\frac{b}{\theta}\right)-\frac{x v^{y}}{\theta}\right\} e^{\theta\left(T-T_{1}\right)}-a \beta c_{p} e^{\theta\left(T-T_{1}\right)}=0 .
\end{aligned}
$$


By solving the equations, one can obtain optimal values of $T_{1}$ and $T$. Using the above theorem,

$$
\frac{\partial^{2} W_{2}\left(T_{1}, T\right)}{\partial^{2} T_{1}}<0, \quad \text { and } \quad\left|\begin{array}{ll}
\frac{\partial^{2} W_{2}\left(T_{1}, T\right)}{\partial T_{1}^{2}} & \frac{\partial^{2} W_{2}\left(T_{1}, T\right)}{\partial T_{1} \partial T} \\
\frac{\partial^{2} W_{2}\left(T_{1}, T\right)}{\partial T_{1} \partial T} & \frac{\partial^{2} W_{2}\left(T_{1}, T\right)}{\partial T^{2}}
\end{array}\right|>0
$$

one can obtain the following results

$$
\begin{aligned}
& p I_{e}\left\{-\alpha \beta\left(\frac{2 a T_{1}}{\theta}-\frac{1}{\theta^{2}}+\frac{b}{\theta}\right)+\frac{x v^{y}}{2}\left(\frac{1}{\theta}+1\right)+2 a \beta T_{1}+b \beta\right\} \\
& -p I_{e}\left\{\beta\left(\frac{a T}{\theta}-\frac{1}{\theta^{2}}+\frac{b}{\theta}\right)-\frac{x v^{y}}{\theta}\right\} e^{\theta\left(T-T_{1}\right)} \\
& +p \theta I_{e}\left\{\beta\left(\frac{a T}{\theta}-\frac{1}{\theta^{2}}+\frac{b}{\theta}\right)-\frac{x v^{y}}{\theta}\right\}\left(1+T_{1}\right) e^{\theta\left(T-T_{1}\right)} \\
& -2 p I_{e}\left\{-\alpha \beta\left(\frac{2 a T_{1}}{\theta}-\frac{1}{\theta^{2}}+\frac{b}{\theta}\right)+\frac{x v^{y}}{\theta}\right\}-\frac{a \alpha \beta p I_{e}}{\theta}\left(m-T_{1}\right) \\
& -p I_{e}\left(m-T_{1}\right)\left\{-\alpha \beta\left(\frac{a T_{1}}{\theta}-\frac{1}{\theta^{2}}+\frac{b}{\theta}\right)+\frac{x v^{y}}{\theta}\right\}-p \theta I_{e}\left\{\beta\left(\frac{a T}{\theta}-\frac{1}{\theta^{2}}+\frac{b}{\theta}\right)\right. \\
& \left.-\frac{x v^{y}}{\theta}\right\} e^{\theta\left(T-T_{1}\right)}-p I_{e}\left\{\beta\left(\frac{a T}{\theta}-\frac{1}{\theta^{2}}+\frac{b}{\theta}\right)-\frac{x v^{y}}{\theta}\right\} e^{\theta\left(T-T_{1}\right)} \\
& -p \theta^{2} I_{e}\left(m-T_{1}\right)\left\{\beta\left(\frac{a T}{\theta}-\frac{1}{\theta^{2}}+\frac{b}{\theta}\right)-\frac{x v^{y}}{\theta}\right\} e^{\theta\left(T-T_{1}\right)} a \beta c_{p} \\
& -\theta^{3} c_{p}\left\{\beta\left(\frac{a T}{\theta}-\frac{1}{\theta^{2}}+\frac{b}{\theta}\right)-\frac{x v^{y}}{\theta}\right\} e^{\theta\left(T-T_{1}\right)}=0 \\
& \left(p-c_{p}\right) a \beta+p \theta^{2} I_{e}\left\{\beta\left(\frac{a T}{\theta}-\frac{1}{\theta^{2}}+\frac{b}{\theta}\right)-\frac{x v^{y}}{\theta}\right\}\left\{e^{\theta T}-\left(1+T_{1}\right) e^{\theta\left(T-T_{1}\right)}\right\} \\
& +\frac{a \beta p I_{e}}{\theta}\left\{e^{\theta T}-\left(1+T_{1}\right) e^{\theta\left(T-T_{1}\right)}\right\}+\left(m-T_{1}\right) p \theta^{2} I_{e}\left\{\beta\left(\frac{a T}{\theta}-\frac{1}{\theta^{2}}+\frac{b}{\theta}\right)\right. \\
& \left.-\frac{x v^{y}}{\theta}\right\}\left\{e^{\theta T}-\left(1+T_{1}\right) e^{\theta\left(T-T_{1}\right)}\right\}+2\left(m-T_{1}\right) a \beta p I_{e}\left\{e^{\theta T}-e^{\theta\left(T-T_{1}\right)}\right\} \\
& +\theta^{2}\left(p-c_{p}-c_{h}\right)(1+\alpha)\left\{\beta\left(\frac{a T}{\theta}-\frac{1}{\theta^{2}}+\frac{b}{\theta}\right)+\frac{x v^{y}}{\theta}\right\} e^{\theta T} \\
& +\theta\left(p-c_{p}-c_{h}\right)(1+\alpha) \frac{a \beta}{\theta} e^{\theta T}+\theta\left(p-c_{p}-c_{h}\right)(1+\alpha) a \beta e^{\theta T}+a \beta c_{p} \\
& >\theta\left(p-c_{p}-c_{h}\right)(1+\alpha) \frac{a \beta}{\theta}+\theta^{3} c_{p}\left\{\beta\left(\frac{a T}{\theta}-\frac{1}{\theta^{2}}+\frac{b}{\theta}\right)-\frac{x v^{y}}{\theta}\right\} e^{\theta\left(T-T_{1}\right)} \\
& -2 a \beta \theta c_{p} e^{\theta\left(T-T_{1}\right)}\left(1+m+\theta c_{P}\right) p I_{e}\left\{\beta\left(\frac{a T}{\theta}-\frac{1}{\theta^{2}}+\frac{b}{\theta}\right)-\frac{x v^{y}}{\theta}\right\} e^{\theta\left(T-T_{1}\right)} \\
& +\left(\theta c_{p}-1\right) \frac{a \beta p I_{e}}{\theta} e^{\theta\left(T-T_{1}\right)}>p I_{e}\left\{\beta\left(\frac{a T}{\theta}-\frac{1}{\theta^{2}}+\frac{b}{\theta}\right)-\frac{x v^{y}}{\theta}\right\} e^{\theta T} .
\end{aligned}
$$$$
\text { (ii) }
$$ 


\section{Appendix D.}

Proof. To maximize $Z_{3}\left(T_{1}, T\right)=\frac{1}{T} W_{3}\left(T_{1}, T\right)$, necessary conditions are as $\frac{\partial Z_{3}\left(T_{1}, T\right)}{\partial T_{1}}=0, \frac{\partial Z_{3}\left(T_{1}, T\right)}{\partial T}=0$ and the sufficient condition are

$$
\frac{\partial^{2} W_{3}\left(T_{1}, T\right)}{\partial^{2} T_{1}}<0, \quad \text { and } \quad\left|\begin{array}{ll}
\frac{\partial^{2} W_{3}\left(T_{1}, T\right)}{\partial T_{1}^{2}} & \frac{\partial^{2} W_{3}\left(T_{1}, T\right)}{\partial T_{1} \partial T} \\
\frac{\partial^{2} W_{3}\left(T_{1}, T\right)}{\partial T_{1} \partial T} & \frac{\partial^{2} W_{3}\left(T_{1}, T\right)}{\partial T^{2}}
\end{array}\right|>0
$$

Now, $\frac{\partial Z_{3}\left(T_{1}, T\right)}{\partial T_{1}}=0$ gives

(i)

$$
\begin{aligned}
p I_{e} & \left\{-\alpha \beta\left(\frac{a T_{1}^{2}}{\theta}-\frac{T_{1}}{\theta^{2}}+\frac{b T_{1}}{\theta}\right)+\frac{x v^{y}}{2} T_{1}\left(\frac{1}{\theta}+1\right)\right. \\
& \left.+a \beta T_{1}^{2}+b \beta T_{1}\right\}-p \theta I_{e}\left\{\beta\left(\frac{a T}{\theta}-\frac{1}{\theta^{2}}+\frac{b}{\theta}\right)-\frac{x v^{y}}{\theta}\right\}\left\{\left(1+T_{1}\right) e^{\theta\left(T-T_{1}\right)}\right\} \\
& -p I_{e}\left\{\beta\left(\frac{a T}{\theta}-\frac{1}{\theta^{2}}+\frac{b}{\theta}\right)-\frac{x v^{y}}{\theta}\right\} e^{\theta\left(T-T_{1}\right)}-p I_{e}\left\{-\alpha \beta\left(\frac{a T_{1}^{2}}{\theta}-\frac{T_{1}}{\theta^{2}}+\frac{b T_{1}}{\theta}\right)\right. \\
& \left.+\frac{x v^{y}}{\theta} T_{1}\right\}+p I_{e}\left(m-T_{1}\right)\left\{-\alpha \beta\left(\frac{a T_{1}}{\theta}-\frac{1}{\theta^{2}}+\frac{b}{\theta}\right)+\frac{x v^{y}}{\theta}\right\} \\
& -p I_{e}\left\{\beta\left(\frac{a T}{\theta}-\frac{1}{\theta^{2}}+\frac{b}{\theta}\right)-\frac{x v^{y}}{\theta}\right\}\left\{e^{\theta T}-e^{\theta\left(T-T_{1}\right)}\right\} \\
& -p \theta I_{e}\left\{\beta\left(\frac{a T}{\theta}-\frac{1}{\theta^{2}}+\frac{b}{\theta}\right)-\frac{x v^{y}}{\theta}\right\} e^{\theta\left(T-T_{1}\right)}-a \beta c_{p} T_{1}+\theta c_{p}\left\{\beta\left(\frac{1}{\theta^{2}}-\frac{b}{\theta}\right)+\frac{x v^{y}}{\theta}\right\} \\
& +\theta^{2} c_{p}\left\{\beta\left(\frac{a T}{\theta}-\frac{1}{\theta^{2}}+\frac{b}{\theta}\right)-\frac{x v^{y}}{\theta}\right\} e^{\theta\left(T-T_{1}\right)}=0 .
\end{aligned}
$$

By solving the equations, one can obtain optimal values of $T_{1}$ and $T$. Using the above theorem,

$$
\frac{\partial^{2} W_{3}\left(T_{1}, T\right)}{\partial^{2} T_{1}}<0, \quad \text { and } \quad\left|\begin{array}{ll}
\frac{\partial^{2} W_{3}\left(T_{1}, T\right)}{\partial T_{1}^{2}} & \frac{\partial^{2} W_{3}\left(T_{1}, T\right)}{\partial T_{1} \partial T} \\
\frac{\partial^{2} W_{3}\left(T_{1}, T\right)}{\partial T_{1} \partial T} & \frac{\partial^{2} W_{3}\left(T_{1}, T\right)}{\partial T^{2}}
\end{array}\right|>0 .
$$

The following results are obtained

$$
\begin{aligned}
p I_{e} & \left\{-\alpha \beta\left(\frac{2 a T_{1}}{\theta}-\frac{1}{\theta^{2}}+\frac{b}{\theta}\right)+\frac{x v^{y}}{2}\left(\frac{1}{\theta}+1\right)+2 a \beta T_{1}+b \beta\right\} \\
& -p I_{e}\left\{\beta\left(\frac{a T}{\theta}-\frac{1}{\theta^{2}}+\frac{b}{\theta}\right)-\frac{x v^{y}}{\theta}\right\} e^{\theta\left(T-T_{1}\right)} \\
& +p \theta I_{e}\left\{\beta\left(\frac{a T}{\theta}-\frac{1}{\theta^{2}}+\frac{b}{\theta}\right)-\frac{x v^{y}}{\theta}\right\}\left(1+T_{1}\right) e^{\theta\left(T-T_{1}\right)} \\
& -2 p I_{e}\left\{-\alpha \beta\left(\frac{2 a T_{1}}{\theta}-\frac{1}{\theta^{2}}+\frac{b}{\theta}\right)+\frac{x v^{y}}{\theta}\right\}-\frac{a \alpha \beta p I_{e}}{\theta}\left(m-T_{1}\right) \\
& -p I_{e}\left(m-T_{1}\right)\left\{-\alpha \beta\left(\frac{a T_{1}}{\theta}-\frac{1}{\theta^{2}}+\frac{b}{\theta}\right)+\frac{x v^{y}}{\theta}\right\}-p \theta I_{e}\left\{\beta\left(\frac{a T}{\theta}-\frac{1}{\theta^{2}}+\frac{b}{\theta}\right)\right. \\
& \left.-\frac{x v^{y}}{\theta}\right\} e^{\theta\left(T-T_{1}\right)}-p I_{e}\left\{\beta\left(\frac{a T}{\theta}-\frac{1}{\theta^{2}}+\frac{b}{\theta}\right)-\frac{x v^{y}}{\theta}\right\} e^{\theta\left(T-T_{1}\right)} \\
& -p \theta^{2} I_{e}\left(m-T_{1}\right)\left\{\beta\left(\frac{a T}{\theta}-\frac{1}{\theta^{2}}+\frac{b}{\theta}\right)-\frac{x v^{y}}{\theta}\right\} e^{\theta\left(T-T_{1}\right)} a \beta c_{p}
\end{aligned}
$$




$$
\begin{aligned}
& -\theta^{3} c_{p}\left\{\beta\left(\frac{a T}{\theta}-\frac{1}{\theta^{2}}+\frac{b}{\theta}\right)-\frac{x v^{y}}{\theta}\right\} e^{\theta\left(T-T_{1}\right)}=0 \\
(p & \left.-c_{p}\right) a \beta+p \theta^{2} I_{e}\left\{\beta\left(\frac{a T}{\theta}-\frac{1}{\theta^{2}}+\frac{b}{\theta}\right)-\frac{x v^{y}}{\theta}\right\}\left\{e^{\theta T}-\left(1+T_{1}\right) e^{\theta\left(T-T_{1}\right)}\right\} \\
& +\frac{a \beta p I_{e}}{\theta}\left\{e^{\theta T}-\left(1+T_{1}\right) e^{\theta\left(T-T_{1}\right)}\right\}+\left(m-T_{1}\right) p \theta^{2} I_{e}\left\{\beta\left(\frac{a T}{\theta}-\frac{1}{\theta^{2}}+\frac{b}{\theta}\right)\right. \\
& \left.-\frac{x v^{y}}{\theta}\right\}\left\{e^{\theta T}-\left(1+T_{1}\right) e^{\theta\left(T-T_{1}\right)}\right\}+2\left(m-T_{1}\right) a \beta p I_{e}\left\{e^{\theta T}-e^{\theta\left(T-T_{1}\right)}\right\} \\
& +\theta^{2}\left(p-c_{p}-c_{h}\right)(1+\alpha)\left\{\beta\left(\frac{a T}{\theta}-\frac{1}{\theta^{2}}+\frac{b}{\theta}\right)+\frac{x v^{y}}{\theta}\right\} e^{\theta T} \\
& +\theta\left(p-c_{p}-c_{h}\right)(1+\alpha) \frac{a \beta}{\theta} e^{\theta T}+\theta\left(p-c_{p}-c_{h}\right)(1+\alpha) a \beta e^{\theta T}+a \beta c_{p} \\
& >\theta\left(p-c_{p}-c_{h}\right)(1+\alpha) \frac{a \beta}{\theta}+\theta^{3} c_{p}\left\{\beta\left(\frac{a T}{\theta}-\frac{1}{\theta^{2}}+\frac{b}{\theta}\right)-\frac{x v^{y}}{\theta}\right\} e^{\theta\left(T-T_{1}\right)} \\
& -2 a \beta \theta c_{p} e^{\theta\left(T-T_{1}\right)} \\
(1+ & \left.+m+\theta c_{P}\right) p I_{e}\left\{\beta\left(\frac{a T}{\theta}-\frac{1}{\theta^{2}}+\frac{b}{\theta}\right)-\frac{x v^{y}}{\theta}\right\} e^{\theta\left(T-T_{1}\right)} \\
& +\left(\theta c_{p}-1\right) \frac{a \beta p I_{e}}{\theta} e^{\theta\left(T-T_{1}\right)}>p I_{e}\left\{\beta\left(\frac{a T}{\theta}-\frac{1}{\theta^{2}}+\frac{b}{\theta}\right)-\frac{x v^{y}}{\theta}\right\} e^{\theta T} .
\end{aligned}
$$

Acknowledgements. This work is supported by the National Research Foundation of Korea (NRF) grant funded by the Korea government (MSIT) (NRF - 2020R1F1A1064460).

\section{REFERENCES}

[1] W. Ahmed and B. Sarkar, Impact of carbon emissions in a sustainable supply chain management for a second generation biofuel. J. Cleaner Prod. 186 (2018) 807-820.

[2] W. Ahmed and B. Sarkar, Management of next-generation energy using a triple bottom line approach under a supply chain framework. Res. Conserv. Recycl. 150 (2019) 104431.

[3] S.X. Bai and S. Varanasi, An optimal production flow control problem with piecewise constant demand. Math. Comput. Mod. 24 (1996) 87-107.

[4] C.T. Chang, L.Y. Ouyang and J.T. Teng, An EOQ model for deteriorating items under supplier credits linked to ordering quantity. Appl. Math. Model. 27 (2003) 983-996.

[5] S.H. Chen, L.E. Cárdenas-Barrón and J.T. Teng, Retailer's economic order quantity when the supplier offers conditionally permissible delay-in-payments link to order quantity. Int. J. Prod. Econ. 155 (2014) 284-291.

[6] S.K. Choi, K.E. Lim and E.Y. Lee, A partial replenishment model for an inventory with constant demand. App. Math. Mod. 32 (2008) 1790-1796.

[7] K.J. Chung, Comments on the EOQ model under retailer partial trade-credit policy in the supply chain. Int. J. Prod. Econ. 114 (2008) 308-312.

[8] B.K. Dey, B. Sarkar, M. Sarkar and S. Pareek, An integrated inventory model involving discrete setup cost reduction, variable safety factor, selling-price dependent demand, and investment. RAIRO: OR 53 (2018) 39-57.

[9] B.K. Dey, B. Sarkar and S. Pareek, A Two-Echelon supply chain management with setup time and cost reduction, quality improvement and variable production rate. Mathematics $\mathbf{7}$ (2019) 328.

[10] B.K. Dey, S. Pareek, M. Tayyab and B. Sarkar, Autonomation policy to control work-in-process inventory in a smart production system. Int. J. Prod. Res. 59 (2021) 1258-1280.

[11] R. Guchhait, B.K. Dey, S. Bhuniya, B. Ganguly, B. Mandal, R.K. Bachar, B. Sarkar, H.M. Wee and K.S. Chaudhuri, Investment for process quality improvement and setup cost reduction in an imperfect production process with warranty policy and shortages. RAIRO: OR 54 (2020) 251-266.

[12] M.W. Iqbal and B. Sarkar, Application of preservation technology for lifetime dependent products in an integrated production system. J. Ind. Manage. Opt. 16 (2020) 141-167. 
[13] M.A.A. Khan, A.A. Shaikh, G.C. Panda and I. Konstantaras, Cárdenas-Barrón, L. E. The effect of advance payment with discount facility on supply decisions of deteriorating products whose demand is both price and stock dependent. Int. Tran. Opera. Res. 27 (2019) 1343-1367.

[14] M.A.A. Khan, A.A. Shaikh, G.C. Panda, I. Konstantaras and A.A. Taleizadeh, Inventory system with expiration date: pricing and replenishment decisions. Comput. Ind. Eng. 132 (2019) 232-247.

[15] M.A.A. Khan, A.A. Shaikh, G.C. Panda and I. Konstantaras, Two-warehouse inventory model for deteriorating items with partial backlogging and advance payment scheme. RAIRO: OR 53 (2019) 1691-1708.

[16] I. Khan, J. Jemai, H. Lim and B. Sarkar, Effect of electrical energy on the manufacturing setup cost reduction, transportation discounts, and process quality improvement in a two-echelon supply chain management under service level constraint. Energies $12(2019) 3733$.

[17] A. Khanna, P. Gautam, B. Sarkar and C.K. Jaggi, Integrated vendor-buyer strategies for imperfect production systems with maintenance and warranty policy. RAIRO: OR $\mathbf{5 4}(2020) 435-450$.

[18] A. Khanna, A. Kishore, B. Sarkar and C.K. Jaggi, Inventory and pricing decisions for imperfect quality items with inspection errors, sales returns, and partial backorders under inflation. RAIRO: OR 54 (2020) 287-306.

[19] S. Khanra, B. Mandal and B. Sarkar, An inventory model with time dependent demand and shortages under trade credit policy. Eco. Mod. 35 (2013) 349-355.

[20] M.S. Kim and B. Sarkar, Multi-stage cleaner production process with quality improvement and lead time dependent ordering cost. J. Cleaner Prod. 144 (2017) 572-590.

[21] A.H.M. Mashud, A.A. Khan, M.S. Uddin and M.N. Islam, A non-instantaneous inventory model having different deterioration rates with stock and price dependent demand under partially backlogged shortages. Uncer. Sup. Chain Manage. 6 (2018) 49-64.

[22] A.H.M. Mashud, M.R. Hasan, H.M. Wee and Y. Daryanto, Non-instantaneous deteriorating inventory model under the joined effect of trade-credit, preservation technology and advertisement policy. Kybernetes 49 (2019) 1645-1674.

[23] A.H.M. Mashud, H.M. Wee and C.V. Huang, Preservation technology investment, trade credit and partial backordering model for a non-instantaneous deteriorating inventory. RAIRO: OR 5 (2021) S51-S77.

[24] A.H.M. Mashud, M.S. Uddin and S.S. Sana, A two-level trade-credit approach to an integrated price-sensitive inventory model with shortages. Int. J. App. Comput. Math. 5 (2019) 121.

[25] U. Mishra, J.Z. Wu and B. Sarkar, A sustainable production-inventory model for a controllable carbon emissions rate under shortages. J. Cleaner Prod. 256 (2020) 120268.

[26] I. Moon, E. Shin and B. Sarkar, Min-max distribution free continuous-review model with a service level constraint and variable lead time. App. Math. Comput. 229 (2014) 310-315.

[27] J.S. Noh, J.S. Kim and B. Sarkar, Two-echelon supply chain coordination with advertising demand under Stackelberg game policy. Eur. J. Ind. Eng. 13 (2019) 213-244.

[28] B. Raa and E.H. Aghezzaf, Designing distribution patterns for long-term inventory routing with constant demand rates. Int. J. Prod. Econ. 112 (2008) 255-263.

[29] S.S. Sana and K.S. Chaudhuri, On a volume flexible stock-dependent inventory model. Proc. Nat. Acad. Sci. Ind. 76 (2006) 309-315.

[30] S.S. Sana and K.S. Chaudhuri, An inventory model for stock with advertising sensitive demand. IMA J. Manage. Math. 19 (2008) 51-62.

[31] B. Sarkar, A production-inventory model with probabilistic deterioration in two-echelon supply chain management. App. Math. Model. 37 (2013) 3138-3151.

[32] B. Sarkar and S. Saren, Partial trade-credit policy of retailer with exponentially deteriorating items. Int. J. App. Comput. Math. 3 (2015) 343-368.

[33] B. Sarkar and S. Sarkar, An improved inventory model with partial backlogging, time varying deterioration and stock-dependent demand. Eco. Mod. 30 (2013) 924-932.

[34] M. Sarkar and B. Sarkar, How does an industry reduce waste and consumed energy within a multi-stage smart sustainable biofuel production system? J. Cleaner Prod. 262 (2020) 121200.

[35] B. Sarkar, S. Sana and K.S. Chaudhuri, A stock-dependent inventory model in an imperfect production process. Int. J. Proc. Manage. 3 (2010) 361-378.

[36] B. Sarkar, S. Sana and K.S. Chaudhuri, An inventory model with finite replenishment rate, trade credit policy and pricediscount offer. J. Ind. Eng. 2013 (2013) 1-18.

[37] B. Sarkar, H. Gupta, K. Chaudhuri and S.K. Goyal, An integrated inventory model with variable lead time, defective units and delay-in-payments. Appl. Math. Comput. 237 (2014) 650-658.

[38] B. Sarkar, S. Saren, D. Sinha and S. Hur, Effect of unequal lot sizes, variable setup cost, and carbon emission cost in a supply chain model. Math. Prob. Eng. 2015 (2015) 469486.

[39] B. Sarkar, S. Sana and K.S. Chaudhuri, Controllable lead time, service level constraint, and transportation discounts in a continuous review inventory model. RAIRO: OR 50 (2016) 921-934.

[40] B. Sarkar, A. Majumder, M. Sarkar, B.K. Dey and G. Roy, Two-echelon supply chain model with manufacturing quality improvement and setup cost reduction. J. Ind. Manage. Opt. 13 (2017) 1085-1104.

[41] B. Sarkar, S. Sana and K.S. Chaudhuri, Environmental and economic assessment of closed-loop supply chain with remanufacturing and returnable transport items. Comput. Ind. Eng. 111 (2017) 148-163. 
[42] B. Sarkar, M. Tayyab, N. Kim and M.S. Habib, Optimal production delivery policies for supplier and manufacturer in a constrained closed-loop supply chain for returnable transport packaging through metaheuristic approach. Comput. Ind. Eng. 135 (2019) 987-1003.

[43] B. Sarkar, M. Omair and N.H. Kim, A cooperative advertising collaboration policy in supply chain management under uncertain conditions. App. Soft Comput. 88 (2020) 105984.

[44] B. Sarkar, B.K. Dey, M. Sarkar, S. Hur, B. Mandal and V. Dhaka, Optimal replenishment decision for retailers with variable demand for deteriorating products under a trade-credit policy. RAIRO: OR $\mathbf{5 4}$ (2020) 1685-1701.

[45] N. Saxena, B. Sarkar and S.R. Singh, Selection of remanufacturing/production cycles with an alternative market: a perspective on waste management. J. Cleaner Prod. 245 (2020) 118935.

[46] S.P. Sethi, Dynamic optimal control models in advertising: a survey. Sia. Rev. 19 (1977) 685-725.

[47] A.A. Shaikh, A.H.M. Mashud, M.S. Uddin and A.A.A. Khan, Non-instantaneous deterioration inventory model with price and stock dependent demand for fully backlogged shortages under inflation. Int. J. Bus. Fore. Market. Int. 3 (2017) $152-164$.

[48] A.A. Shaikh, M.A.A. Khan, G.C. Panda and I. Konstantaras, Price discount facility in an EOQ model for deteriorating items with stock'dependent demand and partial backlogging. Int. Trans. Oper. Res. 26 (2019) 1365-1395.

[49] E.A. Silver and R. Peterson, Decision Systems for Inventory Management and Production Planning, 2nd edition. Wiley, New York (1985).

[50] H.N. Soni and K.A. Patel, Optimal strategy for an integrated inventory system involving variable production and defective items under retailer partial trade-credit policy. Dec. Sup. Sys. 54 (2012) 235-247.

[51] A.A. Taleizadeh, B. Sarkar and M. Hasani, Delayed payment policy in multi-product single-machine economic production quality model with repair failure and partial backordering. J. Ind. Manage. Opt. 16 (2020) 1273-1296.

[52] C.S. Tapiero, Optimal advertising and goodwill under uncertainty. Oper. Res. 26 (1978) 450-463.

[53] M. Tayyab, J. Jemai, L. Han and B. Sarkar, A sustainable development framework for a cleaner multi-item multi-stage textile production system with a process improvement initiative. J. Cleaner Prod. 246 (2020) 119055.

[54] S. Tiwari, W. Ahmed and B. Sarkar, Sustainable ordering policies for non-instantaneous deteriorating items under carbon emission and multi-trade-credit-policies. J. Cleaner Prod. 240 (2019) 118-183.

[55] M. Ullah and B. Sarkar, Recovery-channel selection in a hybrid manufacturing-remanufacturing production model with RFID and product quality. Int. J. Prod. Econ. 219 (2020) 360-374. 